\title{
Elongational rheology of polystyrene melts and solutions: Concentration dependence of the interchain tube pressure effect
}

\author{
Narimissa, Esmaeil; Huang, Qian; Wagner, Manfred H.
}

Published in:

Journal of Rheology

Link to article, DOI:

$10.1122 / 1.5100671$

Publication date:

2020

Document Version

Peer reviewed version

Link back to DTU Orbit

Citation (APA):

Narimissa, E., Huang, Q., \& Wagner, M. H. (2020). Elongational rheology of polystyrene melts and solutions:

Concentration dependence of the interchain tube pressure effect. Journal of Rheology, 64(1), 95-110.

https://doi.org/10.1122/1.5100671

\section{General rights}

Copyright and moral rights for the publications made accessible in the public portal are retained by the authors and/or other copyright owners and it is a condition of accessing publications that users recognise and abide by the legal requirements associated with these rights.

- Users may download and print one copy of any publication from the public portal for the purpose of private study or research.

- You may not further distribute the material or use it for any profit-making activity or commercial gain

- You may freely distribute the URL identifying the publication in the public portal

If you believe that this document breaches copyright please contact us providing details, and we will remove access to the work immediately and investigate your claim 
Elongational Rheology of Polystyrene Melts and Solutions: Concentration Dependence of the Interchain Tube Pressure Effect

\author{
Esmaeil Narimissa ${ }^{1,2}$, Qian Huang ${ }^{3}$, and Manfred H. Wagner ${ }^{4}$ \\ ${ }^{1}$ Dept. of Chemical Engineering, Technion-Israel Institute of Technology (IIT), Technion City, \\ Haifa 32 000, Israel \\ 2 Dept. of Chemical Engineering, Guangdong Technion-Israel Institute of Technology \\ (GTIIT), Shantou 515063, China \\ ${ }^{3}$ Dept. of Chemical and Biochemical Engineering, Technical University of Denmark, 2800 \\ Kongens Lyngby, Denmark \\ ${ }^{4}$ Polymer Engineering/Polymer Physics, Berlin Institute of Technology (TU Berlin), Ernst- \\ Reuter-Platz 1, 10587 Berlin, Germany \\ Corresponding Author: esmaeiln@ technion.ac.il
}

\begin{abstract}
The elongational viscosity data of Bach et al. (2003) and Huang et al. (2013, 2015) on monodisperse polystyrene melts and concentrated polystyrene solutions in oligomeric styrene represent a unique benchmark for improving the tube model of Doi and Edwards with respect to its predictive capabilities of nonlinear viscoelasticity and especially chain stretch. By relaxing one of the basic assumptions of the original tube model of Doi and Edwards (1978) $[1,2]$, i.e. the assumption of a constant tube diameter, and assuming that chain stretch is inverse proportional to a deformation-dependent tube diameter, the Extended Interchain Pressure (EIP) theory (Wagner and Rolón-Garrido, 2009a,b [3, 4]) allowed a parameter-free modelling of the elongational viscosity of monodisperse polystyrene melts. Here we demonstrate that when the dependence of the interchain pressure effect on polymer concentration and molar mass of the oligomeric solvent is considered, the EIP model agrees with experimental evidence that at low Weissenberg numbers $W i_{R}=\dot{\varepsilon} \tau_{R}<1$ melts and solutions show extension thinning behaviours, while at $W i_{R} \cong 1$ solutions switch to extensional thickening or show a more or less constant steady-state elongational viscosity, and melts continue with extension thinning behaviour with a scaling of $\eta_{E} \propto W i_{R}^{-0.5}$. We explain quantitatively the effects of molar mass of solvent and of polymer concentration on the elongational viscosity in the investigated concentration range from $10 \%$ to $100 \%$ (melt), based solely on the linear-viscoelastic characterization. For polystyrene dissolved in oligomeric styrene with molar mass larger than a quarter of the entanglement molar mass of the melt, $M_{o s} \geq M_{e m} / 4=3.3 \mathrm{~kg} / \mathrm{mol}$, we predict a universal relation for the steady-state elongational stress at $W i_{R}>>1$, which is independent of polymer concentration and molar mass of the solvent, while the stretch potential of polystyrene dissolved in oligomeric styrene with $M_{o s}<3.3 \mathrm{~kg} / \mathrm{mol}$ increases with decreasing molar mass of solvent and decreasing polymer concentration in agreement with available experimental evidence.
\end{abstract}




\section{Introduction}

As predicted by the original tube theory (Doi and Edwards 1978 [1, 2], 1986 [5]), the linear viscoelasticity (LVE) of monodisperse linear polymer melts and solutions is governed by the number $Z$ of entanglements per chain, and melts and solutions with the same $Z$ demonstrate the same LVE behaviour [6-9], if the relaxation modulus is scaled by the plateau modulus $G_{N}$, and the frequency is scaled by the equilibrium relaxation time $\tau_{e}$ of a tube segment [7]. However, there is a fundamental difference in the nonlinear viscoelastic behaviour of melts and solutions even with the same $Z$ [7-10]: At low elongation rates $\dot{\varepsilon}$, i.e. at Weissenberg numbers $W i_{R}=\dot{\varepsilon} \tau_{R}<1$ (with Rouse time $\tau_{R}=Z^{2} \tau_{e}$ ), melts and solutions show extension thinning behaviours while at $W i_{R} \approx 1$ solutions switch to extensional thickening, and melts continue with extension thinning behaviour. According to the data of Bach et al. (2003) [11], polystyrene (PS) melts show a monotonic decrease in elongational viscosity $\eta_{E}$ even at strain rates larger that the inverse of the Rouse time, $\dot{\varepsilon}>\tau_{R}^{-1}$, while Bhattacharjee et al. (2002) [12] and Ye et al. (2003) [13] report that for PS solutions, the monotonic decrease in $\eta_{E}$ is followed by a sudden increase at $\dot{\varepsilon} \approx \tau_{R}^{-1}$, when chain stretch is supposed to set in. Also, the extension thinning regime in melts demonstrates an elongation-rate scaling of $\eta_{E} \propto W i_{R}^{-0.5}$ in contrast to the prediction of the original tube model (without chain stretch) of $\eta_{E} \propto W i_{R}^{-1}$.

There is the general agreement that the implementation of chain stretch is an essential feature to improve the predictive capabilities of the tube theory in the nonlinear viscoelastic regime, i.e. at $W i_{R}>1$. However, to explain the observed difference in the nonlinear viscoelastic behaviour of polystyrene melts and solutions, several additional physical mechanisms concerning the modelling of chain stretch have been invoked, such as monomeric friction reduction, nematic interaction, and interchain tube pressure.

Ianniruberto and co-workers (2012) [14, 15] and Yaoita et al. (2012) [16] suggested the concept of monomeric friction coefficient reduction due to flow induced monomer coalignment, which has already been remarked in earlier studies [17-20] and investigated by nonequilibrium molecular dynamics simulations [14, 21]. Based on the work of Yaoita et al. (2012) [16], Desai and Larson (2014) [22] tested a flow induced Kuhn segment alignment friction reduction theory to explain the observed discrepancies between melts and solutions. They assumed that friction is dependent on a stretch/orientation parameter, which when included in a specific form in the DEMG model [23], accelerates both the terminal reptation relaxation as well as fast Rouse relaxation processes but does not cause maximal stretch ratio $\lambda_{\max }$ to change as a function of orientation. Therefore, subchains between entanglements in melts show significant stretching and orientation, which causes the local friction to decrease in fast flows. On the other hand, in the case of solutions with high volume fractions of solvent and consequently a high value of the maximum stretch $\lambda_{\max }$, chain stretching outweighs the Kuhn segment orientation effect and viscosity thickening is observed for fast flows.

However, Desai and Larson (2014) [22] already noticed that recent experimental data of Huang et al. (2015) [8] suggest that details of the solvent, specifically its molar mass, can influence the orientation dependence of the friction, and therefore monomeric friction reduction cannot be the sole reason for the observed differences between melt and solutions. Huang and coworkers conducted a series of experiments on polystyrene dissolved in oligomeric styrene with 
the same $Z$ and different polymer concentrations (from $13 \%$ to $100 \%$, i.e. melt) [8] or different oligomeric solvent molar masses $(1 \mathrm{k}, 2 \mathrm{k}$ and $4 \mathrm{k})$ [7]. These results revealed that polymer concentration has an inverse effect on the extension thickening behaviour of the solutions (the more dilute the solution, the stronger the extension thickening behaviour in the nonlinear regime), and the molar mass of the oligomeric styrene solvents directly affects the fastelongational flow behaviour of the solutions when the lower molar mass solvent $(1 \mathrm{k})$ brings about stronger extension thickening than the larger molar mass solvent (4k). Huang et al. (2013) [7] attributed these observations to nematic interactions between polymer-solvent and polymer-polymer molecules, i.e. the alignment of the polymer molecules due to flow is assumed to induce alignment on the neighbouring molecules (solvent or polymer) via nematic interactions. They reported that different methods such as infrared and ultraviolet dichroism [24, 25], molecular dynamics [26], statistical mechanics [27, 28], double-quantum NMR [29] have documented the presence of local correlations in the orientation of neighbouring molecules during elongational flow of polymeric systems. Huang et al. [7] assumed that in the linear regime no nematic effects on the viscoelastic properties are present so that solutions behave identically to polymer melts with the same number of entanglements. However, in the nonlinear regime, nematic effects are assumed to be important, and the rheological behaviour of polystyrene solutions depends on the chemical structure and size of the solvent molecules. Nematic interaction is supposed to weaken or vanish when the length of the oligomeric solvent is reduced to the size of a polystyrene Kuhn segment (with molar mass of the order of 610 $\mathrm{g} / \mathrm{mol}$ [7] or $700 \mathrm{~g} / \mathrm{mol}[30])$.

To explain the elongational viscosity data of Huang et al. [7, 8, 31], Ianniruberto (2015) [32] and Park and Ianniruberto (2017) [33] presented a flow-induced monomeric friction reduction based multi-mode model with the consideration of nematic interactions. They attributed the friction reduction in extensional flow to the anisotropy of the local environment during fast flow, as opposed to shear flow, where the strong chain stretch is balanced by the rotational component of the deformation. According to their analysis, friction reduction must include oligomer-size-dependent orientational coupling between the oligomeric solvent and the polymer molecules, and such nematic-like interactions vanish for the smallest oligomeric solvent investigated. By a suitable choice of the values of 7 nonlinear fitting parameters for each mode of relaxation, the predictions of the model were in "semiquantitative" agreement with the data of melts and solutions $[32,33]$.

Recently, Matsumiya et al. (2018) [34] claimed that friction reduction, speculatively discussed for entangled melts so far, was experimentally confirmed, for the first time, for two unentangled melts, a polystyrene with molecular mass of $27,000 \mathrm{~g} / \mathrm{mol}$ and a poly(p-tert-butylstyrene) with $53,000 \mathrm{~g} / \mathrm{mol}$. For both materials, the steady-state elongational viscosity exhibits strain-rate hardening followed by strain-rate softening with increasing Weissenberg number. As unentangled melts are free from any entanglement effects, they concluded that the hardening is related to the finite extensible nonlinear elasticity (FENE) of the polymer chains, while the softening is caused by the suppression of the FENE effect due to friction reduction of the highly stretched and oriented chains. By use of a modified FENE bead-spring model, the steady-state elongational viscosity data could be modelled excellently when fitting friction reduction in an empirical way to the steady-state data, while the same model failed to describe the transient stress growth and relaxation data on start-up and cessation of fast elongational flow. They speculated that friction reduction may also depend on transient changes of stretch and orientation. This conjecture of Matsumiya et al. (2018) [34] was recently addressed by Ianniruberto et al. (2019) [35] using Brownian dynamics simulation of Fraenkel chains endowed with a decreasing monomeric friction coefficient again fitted empirically to the 
steady-state elongational viscosity data. The model compares favourably with all start-up and most relaxation data of Matsumiya et al. (2018) [34] if a time delay in reducing the monomeric friction coefficient is accounted for in the fastest flows. While Matsumiya et al. (2018) [34] argue that friction reduction is important for the rheology of unentangled as well as entangled polymer melts, recent Molecular Dynamics (MD) simulations of stress relaxation after elongational flow of a highly oriented entangled polymer melt using a Kremer-Grest beadspring model failed to find direct evidence of friction reduction [36].

When modelling the rheology of linear polymers, usually the assumption is made that stretch created by affine deformation of the chain is balanced by a linear spring force [37]. However, this assumption leads to unbounded stretch at Weissenberg number $W i_{R}=\dot{\varepsilon} \tau_{R}=1$ (see e.g. Wagner (2009) [3, 4]) and is not in agreement with the steady-state elongational viscosity data of monodisperse polystyrene melts ([Bach et al. (2003) [11]) which show a continuously decreasing elongational viscosity for $W i_{R}>1$ with a scaling of approximately $\eta_{E} \propto W i_{R}^{-0.5}$. To remedy this divergence, finite extensibility (FENE), convective constraint release [38], friction reduction $[14,15],[16]$ or a combination of these mechanisms were invoked. An alternative concept of improving the original tube model of Doi and Edwards $(1978)[1,2]$ with respect to its predictive capabilities of nonlinear viscoelasticity and especially chain stretch was the assumption of a deformation-dependent tube diameter with chain stretch being inverse proportional to the tube diameter $[39,40]$. At deformation rates larger than the inverse Rouse time, the tube diameter is reduced by the topological constraints and the chain is increasingly stretched. Marrucci and Ianniruberto (2004) [41] proposed an Interchain Pressure (IP) term assuming that the reduction of the tube diameter due to deformation is balanced by the increase of the radial pressure of the confined chain, and introduced a tube diameter relaxation time $\tau_{a}$ . Subsequently, Wagner et al. (2005) [42] developed a constitutive equation combining Rouselike stretch relaxation and tube diameter relaxation (the Extended Interchain Pressure (EIP) model), and showed that in the melt the tube diameter relaxation time can be identified with three times the Rouse time of the chain, $\tau_{a}=3 \tau_{R}[3,4]$. The EIP model predicts quantitatively time-dependent as well as steady-state rheology of nearly monodisperse polymer melts and allows a parameter-free nonlinear viscoelastic modelling of the elongational viscosity, as the Rouse time is fully defined by linear viscoelasticity.

Applying the EIP model to polymer solutions and analysing the data of Huang et al. [7, 8, 31], Wagner (2014) [43, 44] and Wagner et al. (2015) [43, 44] presented scaling relations for linear viscoelasticity and nonlinear elongational viscosity of polystyrene melts and concentrated solutions of polystyrene in oligomeric styrene based exclusively on the relaxation modulus of a reference polymer melt, the volume fraction of polymer in the solution, and the time-molarmass shift as well as the time-temperature shift caused by the reduction of the glass transition temperature $T_{\mathrm{g}}$ of the polymer in a solution relative to $T_{\mathrm{g}}$ of the melt. These scaling relations were also applied to solutions of polystyrene in diethyl phthalate (DEP) investigated by Bhattacharjee et al. (2002) [12] and Acharya et al. (2008) [45], and except for a possible influence of solvent quality, linear and nonlinear viscoelasticity of entangled PS solutions could be obtained from the linear viscoelastic characteristics of a reference polymer melt and the shift of the glass-transition temperature between melt and solution [44]. However, this approach relied on two stretch relaxation times, namely the Rouse time of the solution and the Rouse time of the parent melt.

In this study, we demonstrate that when the dependence of the interchain tube pressure effect on polymer concentration and molar mass of the oligomeric solvent is considered, agreement 
of the EIP model with the elongational benchmark data of Bach et al. (2003) [11] and Huang et al. (2013, 2015) [7-9] as well as the polystyrene solution data of Bhattacharjee et al. (2002) [12] and Acharya et al. (2008) [45] can be obtained in the investigated concentration range from $10 \%$ to $100 \%$ (melt) based solely on the Rouse stretch relaxation time of the solution.

\section{Experimental data and LVE Characterization}

The experimental data of this research are from the series of studies conducted on PS solutions and melts by Huang and co-workers [7-9]. They investigated the elongational viscosity of PS with nominal molar masses of $285,545,900,1760,3280 \mathrm{~kg} / \mathrm{mol}$ dissolved in oligomeric styrene (OS) solvents with different molar masses $(1 \mathrm{k} / 2 \mathrm{k} / 4 \mathrm{k})$ at different concentrations. Also, the PS solution data of Bhattacharjee et al. (2002) [12] and Acharya et al. (2008) [45] with diethyl phthalate (DEP) as solvent are considered (Table I).

From linear-viscoelastic mastercurves of G' and G', continuous Baumgärtel-SchausbergerWinter (BSW) relaxation spectra [46] were determined [7-9]. The relaxation modulus $G(t)$ is found from the spectrum $H(\tau)$ by

$$
G(t)=\int_{0}^{\infty} \frac{H(\tau)}{\tau} \exp (-t / \tau) d \tau
$$

$H(\tau)$ is composed of two part, the entanglement contribution $H_{e}(\tau)$ and the glassy contribution $H_{g}(\tau)$

$$
H(\tau)=H_{e}(\tau)+H_{g}(\tau)
$$

with

$$
H_{e}(\tau)=n_{e} G_{N}\left(\frac{\tau}{\tau_{t}}\right)^{n_{e}} h\left(1-\tau / \tau_{t}\right)
$$

and

$$
H_{g}(\tau)=n_{e} G_{N}\left(\frac{\tau}{\tau_{c}}\right)^{-n_{g}} h\left(1-\tau / \tau_{t}\right)
$$

$G_{N}$ is the plateau modulus, $\tau_{t}$ the longest or "terminal" relaxation time, $\tau_{c}$ the characteristic time constant of the glassy contribution (related to the equilibrium relaxation time $\tau_{e}$ ), and $h(x)$ the Heaviside step function. The values of $n_{e}$ and $n_{g}$ are fixed to 0.23 and 0.70 , respectively $[7,9]$. The physical meaning of the BSW parameters can be found elsewhere [9]. The zero-shear viscosity of the samples is related to the BSW parameters by

$$
\eta_{0}=\int_{0}^{\infty} G(s) d s=n_{e} G_{N} \tau_{t}\left(\frac{1}{1+n_{e}}+\frac{1}{1-n_{g}}\left(\frac{\tau_{c}}{\tau_{t}}\right)^{n_{g}}\right) \cong n_{e} G_{N} \tau_{t}\left(\frac{1}{1+n_{e}}\right)
$$

As $\tau_{c} \ll<\tau_{t}$, the glassy contribution to $\eta_{0}$ can be neglected. From the plateau modulus of the samples the entanglement molar mass is calculated as [9] 


$$
M_{e}=\frac{\rho R T}{G_{N}} \varphi
$$

where $\rho$ is the density of PS melt at the testing temperature, $R$ is the gas constant, and $\varphi$ is the volume fraction of polymer in the solution with $\varphi=1$ specifying the melt. With $M$ being the molar mass of the polymer, the number of entanglements per chain, $Z$, is obtained,

$$
Z=\frac{M}{M_{e}}
$$

The relation between the entanglement molar mass of solution, $M_{e}$, and melt, $M_{e m}$, is given by

$$
M_{e}=M_{e m} \varphi^{-\alpha}
$$

The value of the dilution exponent $\alpha$ is model dependent $(1<\alpha<4 / 3)$ [12, 47-50], and a value of $\alpha=1$ is taken here [9]. The number of Kuhn segments between entanglements is $N_{e}=M_{e} / M_{0}$, where $M_{0}$ is the molar mass of the Kuhn segment. For polystyrene melt, we take $M_{e m}=13300 \mathrm{~g} / \mathrm{mol}$ [6] and $M_{0}=610 \mathrm{~g} / \mathrm{mol}$ [7], and the value of $M_{0}$ for polystyrene melts and solutions is assumed to be the same [9].

The Rouse stretch relaxation time of solutions $\tau_{R}$ as a function of the testing temperature $T$ (Kelvin) is calculated according to [51-54],

$$
\tau_{R}=\frac{12 M \eta_{0}}{\pi^{2} \rho R T \varphi}\left(\frac{M_{c m}}{M \varphi}\right)^{2.4}
$$

$M_{c m}$ denotes the critical molar mass in the melt state and was taken as $M_{c m}=35000 \mathrm{~g} / \mathrm{mol}[43$, $55,56]$.

The extensional stress measurements of the samples were performed by filament stretching rheometers (FSR). The experimental details of the extensional measurements can be found in [9] and [12]. Tables 1 and 2 show the polymer fraction $(\varphi)$, molar mass $(M)$, polydispersity index (PDI), glass transition temperature $\left(T_{g}\right)$, plateau modulus $\left(G_{N}\right)$, terminal relaxation time $\left(\tau_{t}\right)$, characteristic relaxation time $\left(\tau_{c}\right.$, taken as equivalent of segmental equilibration time $\tau_{e}$ ), zero-shear viscosity $\left(\eta_{0}\right)$, Rouse stretch relaxation time $\left(\tau_{R}\right)$, number of entanglements per chain $(Z)$, and number of Kuhn segments between entanglements $\left(N_{e}\right)$. 
Table 1. Components, weight percentage, molar mass, polydispersity index, and glass transition temperature of polystyrene melts and solutions, oligomeric styrenes and DEP. Data summarized from [7-9] and [44].

\begin{tabular}{|l|c|c|c|c|}
\hline Sample name & $\varphi$ [wt. \% of PS] & $\boldsymbol{M}$ [g/mol] & PDI & $\boldsymbol{T}_{\boldsymbol{g}}\left[{ }^{\circ} \mathbf{C}\right]$ \\
\hline PS-285k & 100 & 285,000 & 1.09 & 107.5 \\
\hline PS-285k/2k-72 & 72 & 285,000 & 1.09 & 94.0 \\
\hline PS-285/2k-44 & 44 & 285,000 & 1.09 & 85.0 \\
\hline PS-545k & 100 & 545,000 & 1.12 & 106.5 \\
\hline PS-545k/4k-52 & 52 & 545,000 & 1.12 & 98.6 \\
\hline PS-545/2k-58 & 58 & 545,000 & 1.12 & 91.0 \\
\hline PS-545k/1k-52 & 52 & 545,000 & 1.12 & 54.0 \\
\hline PS-900k/4k-33 & 33 & 910,000 & 1.16 & 92.1 \\
\hline PS-1760k/4k-18 & 18 & $1,860,000$ & 1.13 & 89.0 \\
\hline PS-3280k/4k-13 & 13 & $3,140,000$ & 1.38 & 88.4 \\
\hline 1k & 0 & 972 & 1.12 & - \\
\hline 2k & 0 & 1920 & 1.08 & 60.5 \\
\hline 4k & 0 & 3630 & 1.06 & - \\
\hline PS-1950/DEP-10 & 10 & $1,950,000$ & - & - \\
\hline PS-1950/DEP-15 & 15 & $1,950,000$ & - & - \\
\hline PS-1950/DEP-20 & 20 & $1,950,000$ & - & - \\
\hline PS-3900/DEP-10 & 10 & $3,900,000$ & 1.05 & - \\
\hline PS-3900/DEP-10 & 15 & $3,900,000$ & 1.05 & - \\
\hline PS-3900/DEP-10 & 17 & $3,900,000$ & 1,05 & - \\
\hline DEP & 0 & 222 & 1 & - \\
\hline
\end{tabular}

Table 2. Material properties of polystyrene melts and solutions in oligomeric styrene at $130^{\circ} \mathrm{C}$ as reported in [7-9], and solutions in DEP at $21.5^{\circ} \mathrm{C}$ as reported in [44].

\begin{tabular}{|l|l|l|c|l|c|c|c|}
\hline Sample name & $\boldsymbol{G}_{N}[\mathbf{P a}]$ & $\tau_{t}[\mathbf{s}]$ & $\tau_{\boldsymbol{c}}[\mathbf{s}]$ & $\eta_{0}[\mathbf{P a} \cdot \mathbf{s}]$ & $\tau_{\boldsymbol{R}}[\mathbf{s}]$ & $\boldsymbol{Z}$ & $\boldsymbol{N}_{\boldsymbol{e}}$ \\
\hline PS-285k & $2.52 \times 10^{5}$ & 6846 & 0.444 & $3.242 \times 10^{8}$ & 216.3 & 21.4 & 22.1 \\
\hline PS-285k/2k-72 & $1.33 \times 10^{5}$ & 381.5 & 0.0736 & $9.613 \times 10^{6}$ & 19.6 & 15.4 & 30.7 \\
\hline PS-285/2k-44 & $4.57 \times 10^{4}$ & 24.29 & 0.0268 & $2.145 \times 10^{5}$ & 2.33 & 9.4 & 50.2 \\
\hline PS-545k & $2.57 \times 10^{5}$ & 58750 & 0.419 & $2.823 \times 10^{9}$ & 776.8 & 41.0 & 22.1 \\
\hline PS-545k/4k-52 & $6.89 \times 10^{4}$ & 3180 & 0.200 & $4.097 \times 10^{7}$ & 92.5 & 21.6 & 42.3 \\
\hline PS-545/2k-58 & $8.41 \times 10^{4}$ & 1112 & 0.0513 & $1.756 \times 10^{7}$ & 30.2 & 23.8 & 38.1 \\
\hline PS-545k/1k-52 & $6.76 \times 10^{4}$ & 9.85 & $5.7 \times 10^{-4}$ & $1.245 \times 10^{5}$ & 0.26 & 21.6 & 42.3 \\
\hline PS-900k/4k-33 & $2.73 \times 10^{4}$ & 5844 & 0.320 & $2.839 \times 10^{7}$ & 163.5 & 22.5 & 67.2 \\
\hline PS-1760k/4k-18 & $6.85 \times 10^{3}$ & 12615 & 0.661 & $1.537 \times 10^{7}$ & 251.7 & 22.8 & 123.2 \\
\hline PS-3280k/4k-13 & $2.79 \times 10^{3}$ & 26373 & 1.511 & $1.619 \times 10^{7}$ & 390.9 & 22.2 & 169.0 \\
\hline PS-1950/DEP-10 & $2.09 \times 10^{3}$ & 0.94 & $2.2 \times 10^{-4}$ & $3.68 \times 10^{2}$ & 0.06 & 14.7 & 217.5 \\
\hline PS-1950/DEP-15 & $4.69 \times 10^{3}$ & 5.0 & $3.0 \times 10^{-4}$ & $3.39 \times 10^{3}$ & 0.07 & 22.8 & 140.2 \\
\hline PS-1950/DEP-20 & $8.35 \times 10^{3}$ & 10.5 & $2.3 \times 10^{-4}$ & $1.56 \times 10^{4}$ & 0.08 & 30.4 & 105.2 \\
\hline PS-3900/DEP-10 & $2.09 \times 10^{3}$ & 11.3 & $2.5 \times 10^{-4}$ & $4.42 \times 10^{3}$ & 0.13 & 30.5 & 209.6 \\
\hline PS-3900/DEP-15 & $4.69 \times 10^{3}$ & 51.6 & $2.9 \times 10^{-4}$ & $4.53 \times 10^{4}$ & 0.20 & 45.7 & 139.9 \\
\hline PS-3900/DEP-17 & $6.03 \times 10^{3}$ & 105 & $3.9 \times 10^{-4}$ & $1.19 \times 10^{5}$ & 0.33 & 51.7 & 123.7 \\
\hline
\end{tabular}




\section{The extended interchain pressure (EIP) model}

The tube model of Doi and Edwards (DE) (1986) [5] assumes that the diameter of the tube $a_{0}$ is constant, i.e. the tension in the macromolecular chain remains constant and equal to its equilibrium value even for nonlinear deformations. The extra stress tensor $\sigma(t)$ is then the consequence of the orientation of tube segments due to the flow. The resulting constitutive equation is of the single integral form, if the tube segments are assumed to align independently of each other in the flow field (i.e. the "Independent Alignment (IA)" approximation),

$$
\sigma(t)=\int_{-\infty}^{t} \frac{\partial G\left(t-t^{\prime}\right)}{\partial t^{\prime}} \mathbf{S}_{\mathrm{DE}}^{\mathrm{IA}}\left(t, t^{\prime}\right) d t^{\prime}
$$

$G(t)$ is the linear-viscoelastic shear relaxation modulus, and the relative strain measure $\mathbf{S}_{\mathrm{DE}}^{\mathrm{IA}}$ is given by

$$
\mathbf{S}_{\mathrm{DE}}^{\mathrm{IA}}\left(t, t^{\prime}\right) \equiv 5\left\langle\frac{\mathbf{u}^{\prime} \mathbf{u}^{\prime}}{u^{\prime 2}}\right\rangle_{\mathrm{o}}=5 \mathbf{S}\left(t, t^{\prime}\right)
$$

$\mathbf{S}$ is the relative second order orientation tensor. The bracket denotes an average over an isotropic distribution of unit vectors $\mathbf{u}\left(t^{\prime}\right)$ at time $t^{\prime}$, and can be expressed as a surface integral over the unit sphere,

$$
\langle\rangle_{\mathrm{o}} \equiv \frac{1}{4 \pi} \mathbb{\int} \int[] \sin \theta_{o} d \theta_{o} d \phi_{o}
$$

At the observation time $t$, the unit vectors are deformed to vectors $\mathbf{u}^{\prime}$, which are calculated from the affine deformation hypothesis (with $\mathbf{F}^{-1}\left(t, t^{\prime}\right)$ being the relative deformation gradient tensor) as

$$
\mathbf{u}^{\prime}\left(t, t^{\prime}\right)=\mathbf{F}^{-1}\left(t, t^{\prime}\right) \cdot \mathbf{u}\left(t^{\prime}\right)
$$

$u^{\prime}$ indicates the length of the vector $\mathbf{u}^{\prime}$.

The DE model does not account for any strain hardening. Doi and Edwards (1986) [5] added a stretch process with a stretch $\lambda$ of the tube segments to their model in order to explain the discrepancies of the DE theory at start-up of extensional flows. Pre-averaging the stretch, i.e. assuming that the stretch is uniform along the chain contour length and an explicit function of the observation time $\lambda(t)$, the extra stress tensor is given by

$$
\sigma(t)=\lambda^{2}(t) \int_{-\infty}^{t} \frac{\partial G\left(t-t^{\prime}\right)}{\partial t^{\prime}} \mathbf{S}_{\mathrm{DE}}^{\mathrm{IA}}\left(t, t^{\prime}\right) d t^{\prime}
$$

Eq. (14) required finding a stretch evolution equation, and a vast variety of concepts based on different kinetic ideas have been proposed in recent years [see e.g. Doi and Edwards(1986) [5], Pearson et al. (1989) [37], McLeish and Larson (1998) [57], and Mead et al. (1998) [58]]. However, it should be noted that Eq. (14) with any function $\lambda^{2}(t)$ is not in agreement with 
experimental results of reversed elongational flow of a monodisperse polystyrene melt (Nielsen et al. (2008) [59]).

While in models with pre-averaged stretch, the tube diameter is always assumed to stay constant and equal to its equilibrium value $a_{0}$, stretch can also be introduced by the assumption of a strain-dependent tube diameter, as first suggested by Marrucci and de Cindio (1980) [60]. Accordingly, the pre-averaging of the stretch can be avoided as well, which is inherently present in models based on Eq. (14) or its differential approximations. A generalized tube model with strain-dependent tube diameter was presented by Wagner and Schaeffer [(1992) [61], (1993) [62] and (1994)] [63], and Wagner et al. (2001) [64]. In the Molecular Stress Function (MSF) theory, tube segment stretch $f=f\left(t, t^{\prime}\right)$ is the inverse of the relative tube diameter $a$,

$$
f\left(t, t^{\prime}\right)=a_{0} / a\left(t, t^{\prime}\right)
$$

which decreases from its equilibrium value $a_{0}$ with increasing stretch. Considering that the tube diameter $a$ represents the mean field of the surrounding chains, it is assumed that the tube diameter is independent of tube segment orientation. The extra stress is then given as

$$
\sigma(t)=\int_{-\infty}^{t} \frac{\partial G\left(t-t^{\prime}\right)}{\partial t^{\prime}} f^{2} \mathbf{S}_{D E}^{I A}\left(t, t^{\prime}\right) d t^{\prime}
$$

In contrast to Eq. (14), stretch in Eq. (16) does not only depend on the observation time $t$, but depends on the strain history: for time-dependent strain histories, chain segments with long relaxation times (i.e. at the centre of the chain) experience higher stretches than chain segments with short relaxation times (i.e. at the chain ends).

Based on the "interchain tube pressure" concept of Marrucci and Ianniruberto (2004) [41], Wagner and Rolón-Garrído (2009a,b) [3, 4] developed an Extended Interchain Pressure (EIP) model consisting of Eq. (16) and an evolution equation for the molecular stretch $f$,

$$
\frac{\partial f}{\partial t}=f(\kappa: \mathbf{S})-\left(1-2 \frac{\tau_{R}}{\tau_{a}}\right) \frac{f-1}{\tau_{R}}-\frac{2}{3} \frac{f^{2}\left(f^{3}-1\right)}{\tau_{a}}
$$

with the initial conditions $f\left(t=t^{\prime}, t^{\prime}\right)=1$. The first term on the right hand side represents an on average affine stretch rate with $\kappa$ the velocity gradient tensor, the second term takes into account Rouse relaxation with Rouse time $\tau_{R}$ according to Eq. (9) in the longitudinal direction of the tube, and the third term limits molecular stretch due to the interchain tube pressure in the lateral direction of a tube segment with tube diameter relaxation time $\tau_{a}$. It should be noted that in the limit of small stretch, i.e. for $f-1<<1$, the interchain pressure term reduces to $f^{2}\left(f^{3}-1\right) \cong 3(f-1)$ and the classical relation of Pearson et al. (1989) [37] is recovered from Eq. (17),

$$
\frac{\partial f}{\partial t}=f(\kappa: \mathbf{S})-\frac{1}{\tau_{R}}(f-1)
$$


According to the analysis of Marrucci and Ianniruberto (2004) [41] (their Eq. (19) within a numerical factor of 3$), \tau_{a}$ is expected to be proportional to the Rouse relaxation time $\tau_{R}$,

$$
\tau_{a}=3 \frac{a_{0}^{4}}{l^{4}} \tau_{R}
$$

$a_{0}=\sqrt{N_{e} b^{2}}$ is the tube diameter and chain segment length between entanglements containing $N_{e}$ Kuhn monomers of length $b$, and the unknown interaction length $l$ represents the size of a blob of the test chain, which can diffuse in the lateral direction in a time $\tau_{R}$ thereby pushing back the confining chains and dissipating stretch energy of the test chain.

Marrucci and Ianniruberto (2004) [41] expected the interaction length $l$ to fall within the interval $b<l<a_{0}$, and suggested a value of $l=\sqrt{b a_{0}}$. However, by considering the small stretch limit of the interchain pressure effect, Wagner and Rolón-Garrído (2009a,b) [3, 4] realized that in the melt, the tube diameter relaxation time $\tau_{a}$ is equal to three times the Rouse time $\tau_{R}$,

$$
\tau_{a}=3 \tau_{R}
$$

This means that in the melt (designated by index $m$ in the following), the length $l$ of the blob of the test chain is equal to the tube diameter $a_{0 m}=\sqrt{N_{e m} b^{2}}$ of the melt and contains $N_{e m}$ monomers, or equivalently that in the closest possible packing of chains, i.e. in the melt state, a chain segment with $N_{e m}$ monomers can explore a lateral distance of magnitude $a_{0 m}$ in the time span of $\tau_{R}$ in order to push back the confining chains and relax stretch (Fig. 1). Excellent agreement between the EIP model and the elongational viscosity data of the monodisperse melts PS390k and PS200k was shown [3, 4], as well as excellent agreement for melts PS-285k and PS-545k [43, 65] without resort to friction reduction. Particularly, the extension thinning regime of the elongational viscosity with an elongation-rate scaling of $\eta_{E} \propto W i_{R}^{-0.5}$ is quantitatively predicted without any free nonlinear fitting parameter, as the Rouse time $\tau_{R}$ is fully determined by Eq. (9).

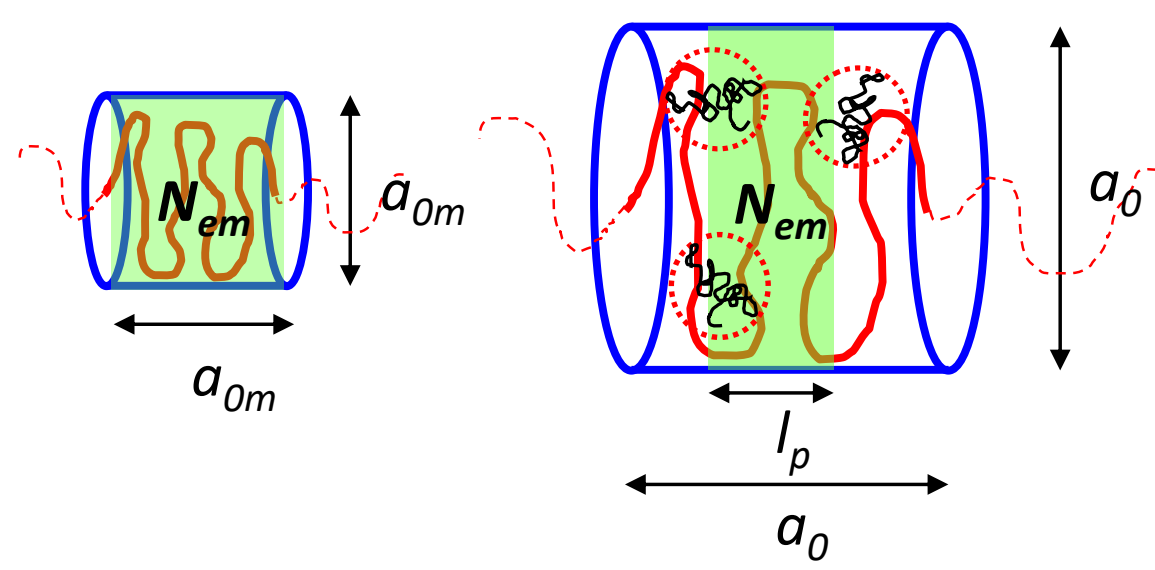


FIG 1. Tube segment of melt (left) and solution with solvent molecules indicated schematically by spheres (right). For details see text.

From the concept of the interchain pressure it is evident that the pressure between the test chain and the confining chains will decrease with dilution as the distance between the chains increases, and the tube diameter relaxation time will increase. Consequently, in dilution, the chains can be stretched much more before the interchain pressure limits further stretch. For polystyrene with volume fraction $\varphi$ dissolved in oligomeric styrene, the number of monomers in a tube segment increases with dilution by $N_{e}=N_{e m} \varphi^{-1}$ according to Eq. (8), and consequently the tube diameter increases with $a_{0}=\sqrt{N_{e} b^{2}}=\sqrt{N_{e m} b^{2}} \varphi^{-1 / 2}=a_{0 m} \varphi^{-1 / 2}$. In solution, the tube of the test chain is filled by the monomers of the test chain, monomers of confining chains, and oligomeric styrene (indicated schematically by spheres in Fig. 1). For the stretch relaxation process, it is necessary that contacts between the test chain and the confining chains take place by lateral fluctuations of the test chain, which permanently push back the confining chains. These contacts can occur directly, i.e. by chain-chain interactions, or indirectly via solvent molecules in contact with the test chain and the confining chains. The inverse of the interaction length $l$ of the interchain pressure in a solution with polymer volume fraction $\varphi$ is therefore expected to be given by the additive contribution of chain-chain and chain-solvent contacts,

$$
\frac{1}{l}=\varphi \frac{1}{l_{p}}+(1-\varphi) \frac{1}{l_{o s}}
$$

$l_{p}$ and $l_{o s}$ are the interaction lengths for chain-chain and chain-solvent contacts, respectively. As the melt is the reference state, we consider in solution the "same" blob of the test chain with $N_{e m}$ monomers as in the melt, but in solution, the blob has now a diameter $a_{0}=a_{0 m} \varphi^{-1 / 2}>a_{0 m}$ , and a length $l_{p}$ smaller than $a_{0}$ (see shaded area in Fig. 1 containing $N_{e m}$ monomers). According to Doi and Edwards (1978) (see Eq. A9 in [1]), the line density $n / l$, i.e. the number $n$ of monomer units of length $b$ that are found per length $l$ of the tube, is a welldefined thermodynamic quantity and is given by

$$
n / l=a_{0} / b^{2}
$$

Thus, for a blob of the test chain with $n=N_{e m}$ monomers, the interaction length $l=l_{p}$ for chain-chain contacts is obtained from Eq. (22) as

$$
l_{p}=\frac{n b^{2}}{a_{0}}=\frac{N_{e m} b^{2}}{a_{0}}=\frac{a_{0 m}^{2}}{a_{0}}=a_{0 m} \varphi^{1 / 2}
$$

The interaction length $l_{p}$ decreases from a value of $a_{0 m}$ in the melt state with decreasing polymer fraction in proportion to $\varphi^{1 / 2}$, which indicates the expected reduction of interchain pressure. 

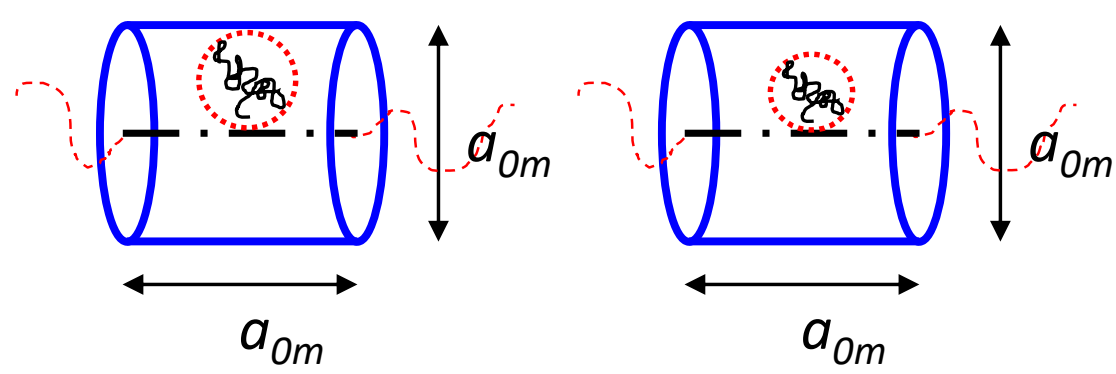

FIG 2. Solvent molecules with diameter equal (left) and less (right) than tube radius. For details see text.

In addition to chain-chain contacts, there are chain-solvent contacts. A solvent molecule, which is temporarily in contact with the test chain and at the same time in contact with one of the confining chains, transmits the lateral fluctuation of the test chain elastically (i.e. as a short entropic spring) to the confining chain, thereby pushing back the confining chain. We note that the reference is again the melt state with a blob of $N_{e m}$ monomers and with tube diameter $a_{0 m}=\sqrt{N_{e m} b^{2}}$. For these chain-solvent contacts, we need to consider the size of the oligomeric styrene in relation to the tube radius. The tube radius of the melt is the relevant measure here, as time-averaged, the test chain is located along the centre axis of the tube segment (the socalled "primitive path"), and the solvent molecules transmit on average the test chain fluctuations laterally from the central axis to the tube boundaries formed by the confining chains. The solvent molecules have a globular shape and are simplistically regarded as spheres (Fig. 2). If the diameter of the solvent molecule is equal to the radius $a_{0 m} / 2$ of the tube, or equivalently if the molar mass $M_{o s}$ of the oligomeric solvent is equal to a quarter of the entanglement molar mass $M_{e m}$, the chain-solvent contacts transmit lateral test chain fluctuations to the confining chains with the same efficiency as chain-chain contacts. The interaction length $l_{o s}$ of the chain-solvent contacts is then equal to the interaction length $l_{p}$ of the chain-chain contacts,

$$
l_{o s}=l_{p}=\frac{n b^{2}}{a_{0}}=\frac{N_{e m} b^{2}}{a_{0}}=a_{0 m} \varphi^{1 / 2}
$$

and the number of monomers of the test chain blob relaxing stretch by chain-solvent interaction is $n=N_{e m}=4 N_{o s}=4 \frac{M_{o s}}{M_{0}}$. Relation (24) will also be valid if the size of the solvent molecule is larger than the radius $a_{0 m} / 2$ of the tube, or equivalently if the molar mass $M_{o s}$ of the solvent is larger than a quarter of the entanglement molar mass $M_{e m}$, as the only condition for the equivalence of $l_{o s}$ and $l_{p}$ is that the size of the solvent molecule is equal or larger than the tube radius. Thus for $M_{o s} \geq M_{e m} / 4$, the lateral transmission of fluctuations of the test chain to the confining chains by oligomeric styrene is as effective as by direct chain-chain interactions. 
If on the other hand the diameter of the solvent molecule is less than the radius $a_{0 m} / 2$ of the tube or equivalently the molar mass of the solvent is less than $M_{e m} / 4$, only a smaller number $n=4 N_{o s}<N_{e m}$ of monomers of the test chain blob can relax stretch by chain fluctuation transfer via chain-solvent contacts to the confining chains, and the interaction length $l_{o s}$ is obtained from Eq. (22) as

$$
l_{o s}=\frac{n b^{2}}{a_{0}}=\frac{4 N_{o s} b^{2}}{a_{0}}=\frac{4 M_{o s}}{M_{e m}} \frac{a_{0 m}^{2}}{a_{0}}=\frac{M_{o s}}{M_{e m} / 4} a_{0 m} \varphi^{1 / 2}
$$

For $M_{o s}<M_{e m} / 4$, the interaction length $l_{o s}$ reduces in proportion to the ratio of $M_{o s}$ to $M_{e m} / 4$, and oligomeric solvents of this type are less effective transmitters of interchain pressure. We remind that the factor $\frac{M_{o s}}{M_{e m} / 4}$ comes from the fact that the relevant length scale for the elastic transmission of test chain fluctuations by solvent molecules is the tube radius, as the oligomeric solvent molecules are not entangled and transmit the stretch relaxation process on average laterally from the central axis of the tube to the tube wall, i.e. on the length scale of the tube radius and not the tube diameter, by having temporary contacts at the same time with both the test chain and one of the confining chains.

From Eqs. (19), (21), and (25), the tube diameter relaxation time is finally obtained as

$$
\tau_{a}=3 \frac{a_{0}^{4}}{l^{4}} \tau_{R}=3 \frac{a_{0 m}^{4}}{l^{4}} \varphi^{-2} \tau_{R}=3 \varphi^{-4}\left[\varphi+(1-\varphi) \frac{M_{e m} / 4}{M_{o s}}\right]^{4} \tau_{R}=3 \Phi^{-4} \tau_{R}
$$

with $\Phi$ being an effective polymer fraction defined for $M_{o s} \leq M_{e m} / 4$ by

$$
\Phi=\frac{\varphi}{\varphi+(1-\varphi) \frac{M_{e m} / 4}{M_{o s}}}
$$

For oligomeric styrene with $M_{o s} \geq M_{e m} / 4$ it follows from Eqs. (21) and (24) that $\Phi$ is identical with the polymer fraction $\varphi$,

$$
\Phi=\varphi
$$

and the tube diameter relaxation time is simply given by

$$
\tau_{a}=3 \frac{a_{0}^{4}}{l^{4}} \tau_{R}=3 \varphi^{-4} \tau_{R}
$$


Thus, for polystyrene dissolved in oligomeric styrene with molar mass of $M_{o s}=\frac{M_{e m}}{4}=\frac{13.3 \mathrm{~kg} / \mathrm{mol}}{4} \cong 3.3 \mathrm{~kg} / \mathrm{mol}$ or larger such as in the case of oligomeric styrene $4 \mathrm{k}$ with $M_{o s}=3.63 \mathrm{~kg} / \mathrm{mol}$ (Table 1 ), the tube diameter relaxation time $\tau_{a}$ is proportional to the inverse $4^{\text {th }}$ power of the polymer fraction $\varphi$.

From Eqs. (17) and (26), the stretch evolution equation is obtained as

$$
\frac{\partial f}{\partial t}=f(\kappa: \mathbf{S})-\left(1-\frac{2}{3} \Phi^{4}\right) \frac{f-1}{\tau_{R}}-\frac{2}{3} \Phi^{4} \frac{f^{2}\left(f^{3}-1\right)}{3 \tau_{R}}
$$

For steady-state elongational flow $\left(\frac{\partial f}{\partial t}=0\right)$ and large stretch, $f^{2}$ reaches a limiting value of

$$
f^{2} \cong \frac{3}{2} \Phi^{-2} \sqrt{2 \tau_{R} \dot{\varepsilon}}
$$

As $f$ is inverse proportional to the tube diameter $a$, it follows from Eq. (31) that the tube diameter $a$ decreases with Weissenberg number $W i_{R}=\dot{\varepsilon} \tau_{R}$ according to $a \propto W i_{R}^{-1 / 4}$. This agrees with recent results of Molecular Dynamics (MD) melt simulations of elongational flow using a Kremer-Grest bead-spring model and taking maximum lateral chain fluctuations as a measure of the tube diameter [66].

From Eqs. (16) and (31), the steady-state elongational stress $\sigma_{E}(\dot{\varepsilon})$ is given at sufficiently large elongation rates, i.e. for $W i_{R}>>1$, by

$$
\sigma_{E}(\dot{\varepsilon})=5 G_{N} f^{2}=\frac{15}{2} G_{N} \Phi^{-2} \sqrt{2 \tau_{R} \dot{\varepsilon}}
$$

Considering that the plateau modulus $G_{N}$ decreases with decreasing polymer fraction according to $G_{N}=G_{N m} \varphi^{2}$, Eq. (32) reveals that for PS dissolved in oligomeric styrene with molar mass equal or larger than $3.3 \mathrm{~kg} / \mathrm{mol}$, i.e. for $\Phi=\varphi$, the steady-state elongational stress $\sigma_{E}$ of polymer solutions is identical to $\sigma_{E}$ of the parent melt at $W i_{R} \gg>1$,

$$
\sigma_{E}(\dot{\varepsilon})=\frac{15}{2} G_{N m} \sqrt{2 \tau_{R} \dot{\varepsilon}}
$$

with $G_{N m}$ being the plateau modulus of the melt. Thus, polymer concentration and molar mass of the solvent (if equal or larger than $3.3 \mathrm{~kg} / \mathrm{mol}$ ) have no effect on the steady-state elongational stress $\sigma_{E}$ at iso- $\mathrm{T}_{\mathrm{g}}$ conditions. Regarding the insensitivity with respect to the molar mass of the solvent, this "universal relation" agrees well with recent data of Shahid et al. [67] on blends of $10 \%$ of a high molar mass polystyrene PS 820 (monodisperse PS with $820 \mathrm{~kg} / \mathrm{mol}$ ) with $90 \%$ matrix styrene with molar masses of $9 \mathrm{~kg} / \mathrm{mol}$ (oligomeric styrene), 23 and $34 \mathrm{~kg} / \mathrm{mol}$ 
(marginally entangled PS), and $73 \mathrm{~kg} / \mathrm{mol}$ (well entangled PS), respectively, which all show the same steady-state elongational viscosity at sufficiently large $\dot{\varepsilon}$. Therefore, even in bidisperse melts, if the difference of the molar masses of the two components is large enough such that the Rouse time of the low molar mass component is much smaller than the Rouse time of the high molar mass component and therefore the low molar mass component remains unstretched, the steady-state elongational stress $\sigma_{E}$ is fully determined by the high molar mass component, irrespective of the molar mass of the polymeric matrix.

The same behaviour was observed earlier by Nielsen et al. (2008) [59] (see also Wagner et al. (2008) [68]) for two blends of 14\% PS390k (390 kg/mol) with $86 \%$ of PS50k (50 kg/mol) and $86 \%$ of PS100k (100 kg/mol), respectively (Fig. 3). Both blends, PS390k/50k and PS390k/100k, show the same steady-state elongational viscosity at sufficiently large $\dot{\varepsilon}$, which is obviously determined by the stretching of the high molar mass chains of PS390k, while the start-up behaviour is influenced by the different linear-viscoelastic extensional stress growth coefficients of PS50k and PS100k, indicated in Figure 3 by dash-dotted lines. To demonstrate that the steady-state viscosity of the blends at large $\dot{\varepsilon}$ is indeed caused by the stretching of the PS390k chains, Figure. 3 also shows predictions (lines) by Eqs. (9), (16), and (30) of the extensional stress growth coefficient of 14\% PS390k dissolved in a hypothetical zero-viscosity solvent with $\Phi=\varphi$. For these predictions, the BSW spectrum of melt PS390k [11] was scaled according to scaling relations presented by Wagner et al. (2015) [44] to a polymer fraction of $\varphi=0.14$, i.e. $G_{N}=G_{N m} \varphi^{2}, \tau_{t}=\tau_{t m} \varphi^{1.4}, \tau_{c}=\tau_{c m} \varphi^{-2}$. As the hypothetical solvent does not have a viscosity, the predictions indicate the underlying stretch of the PS390k chains in the two blends, which is masked by the extensional stress growth coefficient of PS50k and PS100k at smaller stretches and becomes evident only at larger stretches.

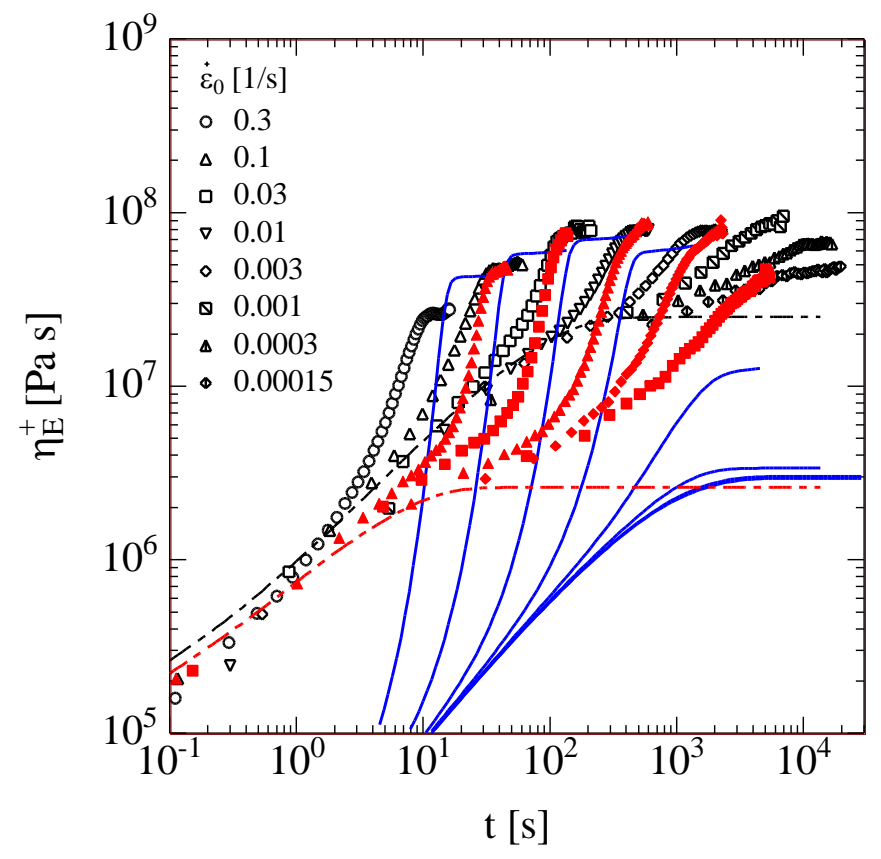


FIG 3. Data of extensional stress growth coefficient of blends of $14 \%$ of PS390k, and $86 \%$ of PS50k (full symbols) and 86\% of PS100k (open symbols), respectively [[59], [68]. Dash-dotted lines indicate linear-viscoelastic extensional stress growth coefficients of PS50k and PS100k. Full lines are predictions for 14\% of PS390k dissolved in hypothetical zero-viscosity solvent according to the EIP model, Eqs. (9), (16), and (30), with $\Phi=\varphi$. For details see text.

In the following section, we assess the modelling competence of the EIP model with evolution equation (30), Rouse stretch relaxation time, Eq. (9), and extra stress tensor, Eq. (16), for the prediction of the elongational viscosity of PS melts and PS dissolved in oligomeric styrene and DEP (Table 1). 


\section{Comparison to experimental data}

\subsection{PS dissolved in 4k oligomeric styrene}

Figures 4 to 7 present comparison of data of the extensional stress growth coefficient and the steady-state elongational viscosity of PS dissolved in oligomeric styrene 4k (Table 1), and predictions by Eqs. (9), (16) and (30) with $\Phi=\varphi$. The steady-state viscosity data of melts PS$545 \mathrm{k}$ and PS-285k and predictions $[43,65]$ are also presented. The elongation rates used for the measurement of the elongational viscosity are given in the original publications of the data and are identical to the strain rates indicated in the plots of the steady-state elongational viscosities. We note that the steady-state elongational viscosity of PS545k/4k-52 and PS900k/4k-33 shows first a decreasing trend with increasing strain rate, and then extension thickening followed by extension thinning. In the case of PS1760k/4k -18 and PS3250k/4k-13, the extension thinning part at high elongation rates is outside the experimentally accessible window of observation, as the solutions show cohesive fracture, indicated in the graphs by open symbols. Cohesive fracture of PS melts and solutions is observed at large stretch $f$ and is discussed in detail in [69]. Considering that predictions are based solely on the linearviscoelastic characterization of the solutions without using any nonlinear fitting parameter agreement between data and predictions can be rated as fair. Deviations between data and predictions are seen at the lower elongation rates for PS545k/4k-52 and PS900k/4k-33, while deviations seen for PS1760/4k-18 and PS3280/4k-13 may be caused by the increased polydispersity of the PS samples (see Table 1).

From Eq. (32) and $\Phi=\varphi$ follows that the steady-state elongational viscosities of all $4 \mathrm{k}$ solutions should coincide with the melt viscosity for $W i_{R}=\dot{\varepsilon} \tau_{R} \gg 1$, if scaled in the form of $\eta_{E} \varphi^{2} /\left(G_{N} \tau_{R}\right)$. This is shown in Figure 8, and indeed agreement at larger Weissenberg numbers is found for all $4 \mathrm{k}$ solutions considered with the rescaled elongational viscosity of melt PS$285 \mathrm{k}$. At Weissenberg numbers $W i_{R}<1$ extension thinning is predicted, which starts when chain segments are first oriented in flow, i.e. when the strain rate reaches the inverse of the longest relaxation time. Extension thickening is observed for the solutions as $W i_{R}$ approaches 1 , followed again by extension thinning for $W i_{R}>>1$ with a slope of approximately $-1 / 2$ as a signature of the stretch-limiting effect of the interchain pressure. In reasonable agreement with experimental data, polymer concentration has an inverse effect on the extension thickening behaviour of the solutions, i.e. the more dilute the solution, the stronger the extension thickening behaviour caused by a reduction of the interchain pressure and an increase of the tube diameter relaxation time according to Eq. (29). In the melt state, there is no extension thickening, but only extension thinning. 

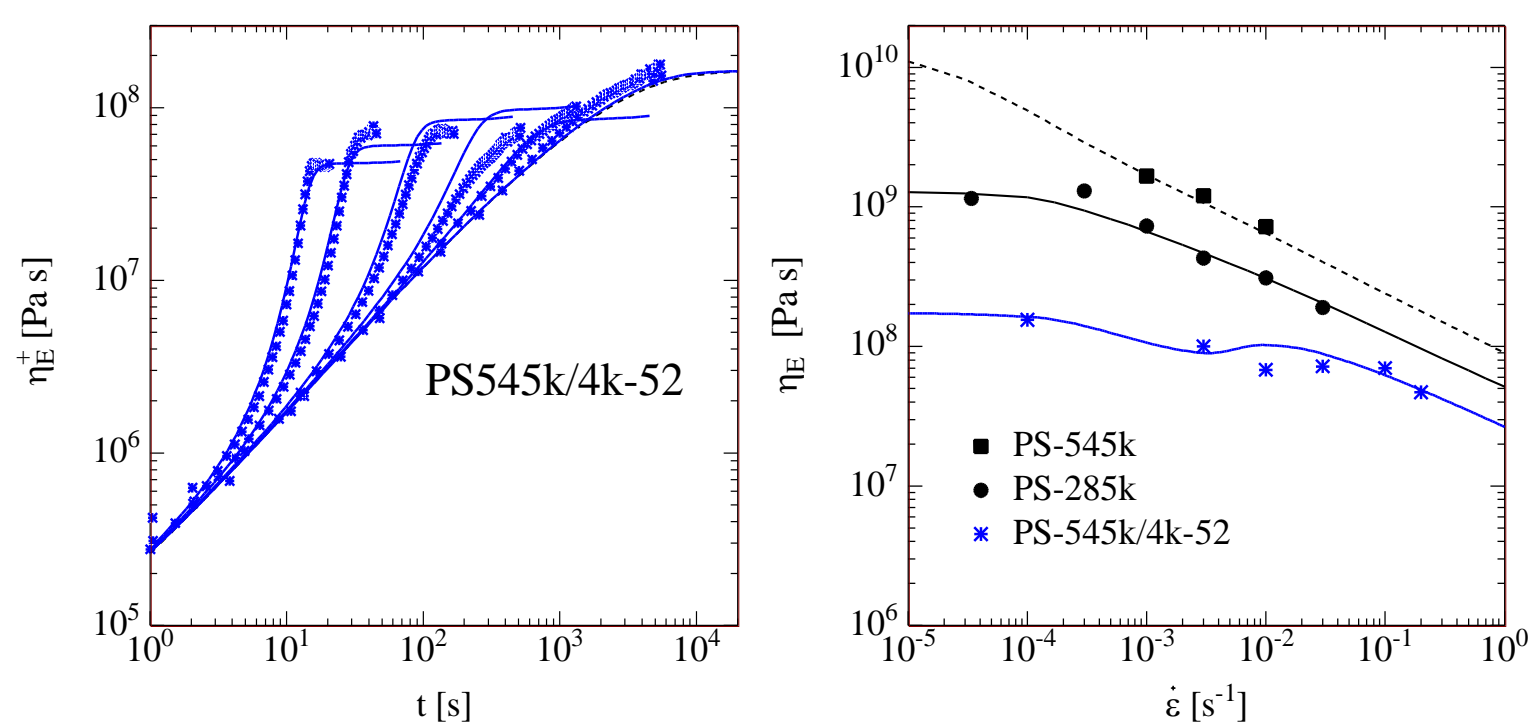

FIG 4. Data of extensional stress growth coefficient (left) and steady-state (right) elongational viscosity (symbols) of 52\% solution PS545k/4k (refer to Table 1) and melts PS-545k and PS285k, and predictions (lines) by EIP model, Eqs. (9), (16), and (30) with $\Phi=\varphi$.
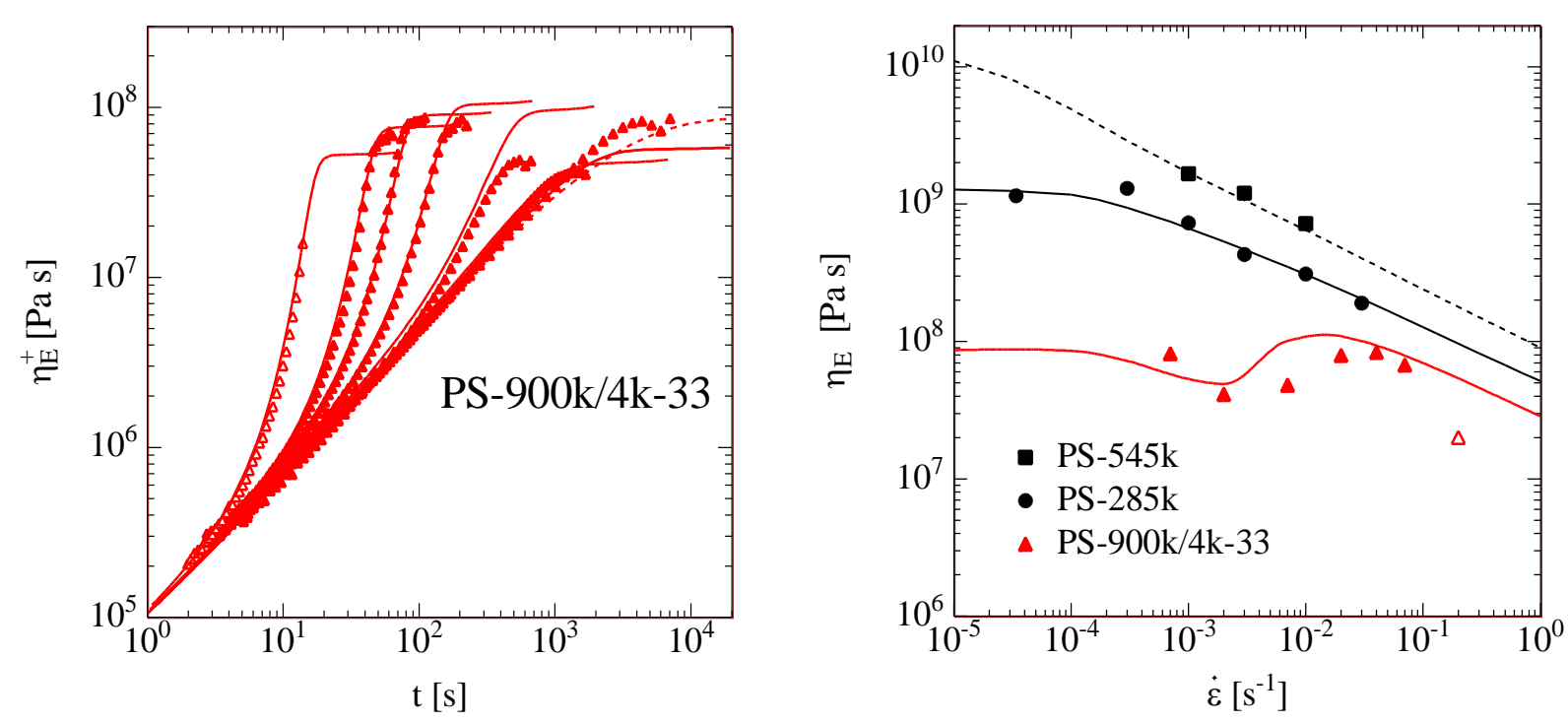

FIG 5. Data of extensional stress growth coefficient (left) and steady-state (right) elongational viscosity (symbols) of $33 \%$ solution PS900k/4k (refer to Table 1) and melts PS-545k and PS285k, and predictions (lines) by EIP model, Eqs. (9), (16), and (30) with $\Phi=\varphi$. Open symbol (right) indicates sample, which fractured at the end of the test. 

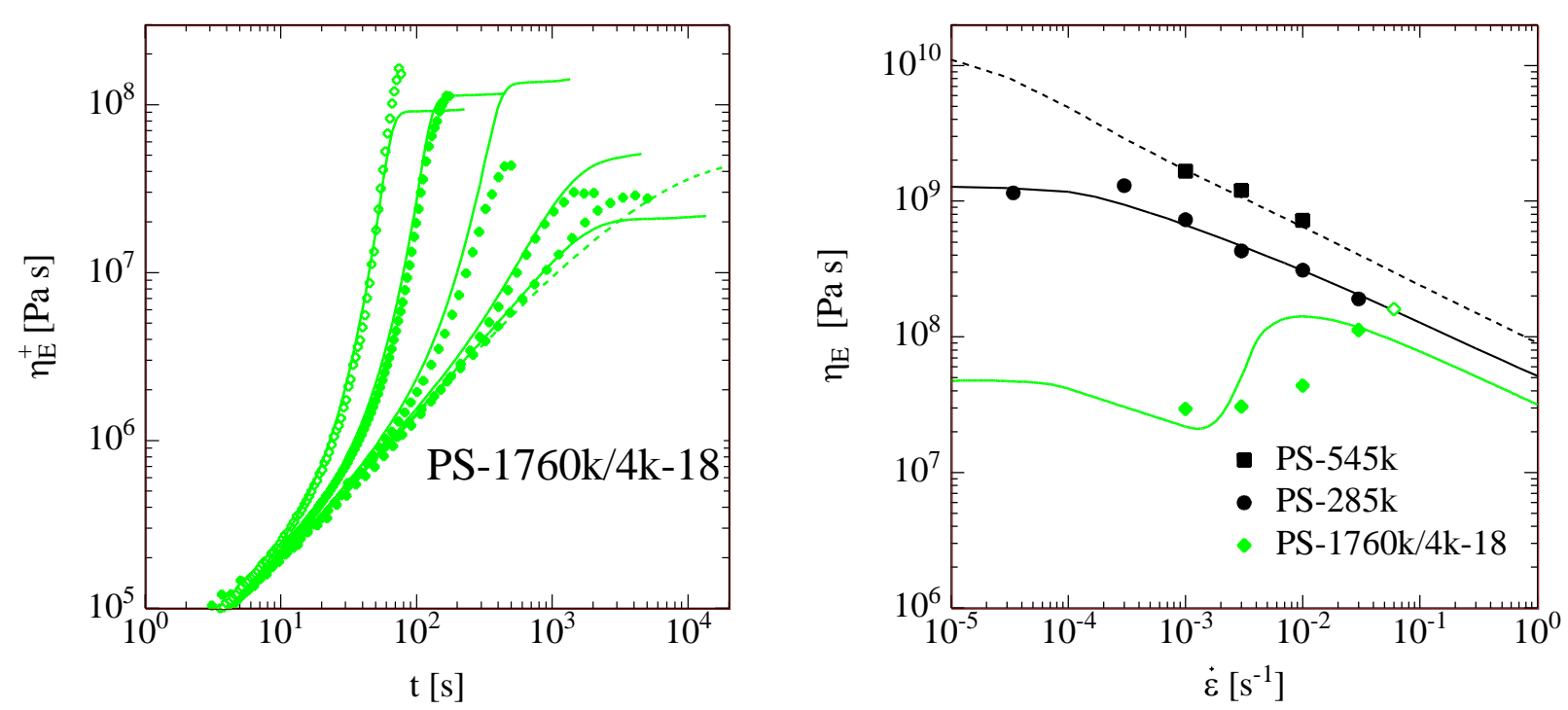

FIG 6. Data of extensional stress growth coefficient (left) and steady-state (right) elongational viscosity (symbols) of $18 \%$ solution PS1760k/4k -18 (refer to Table 1) and melts PS-545k and PS-285k, and predictions (lines) by EIP model, Eqs. (9), (16), and (30) with $\Phi=\varphi$. Open symbol (right) indicates sample, which fractured at the end of the test.
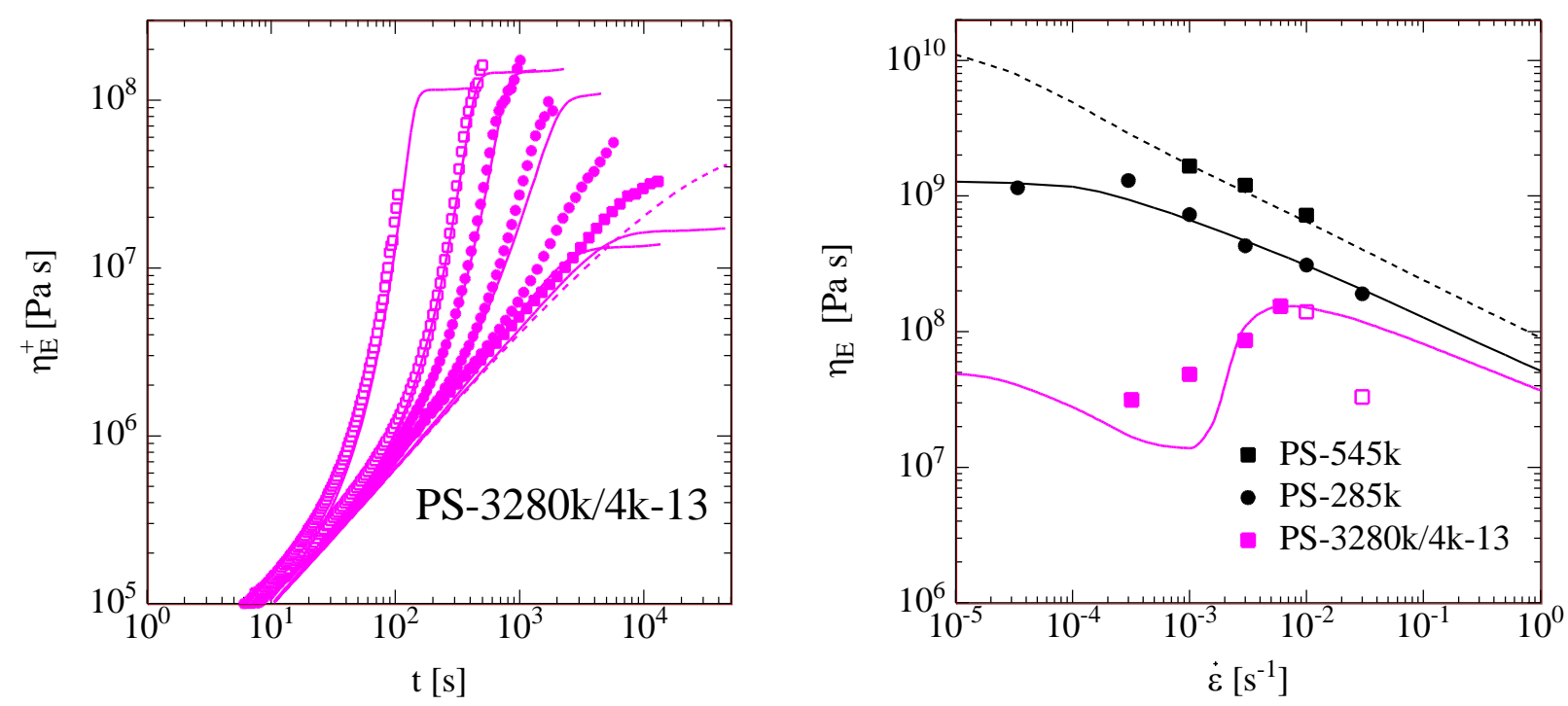

FIG 7. Data of extensional stress growth coefficient (left) and steady-state (right) elongational viscosity (symbols) of $13 \%$ solution PS3280k/4k-13 (refer to Table 1) and melts PS-545k and PS-285k, and predictions (lines) by EIP model, Eqs. (9), (16), and (30) with $\Phi=\varphi$. Open symbols (right) indicate samples, which fractured at the end of the test. 


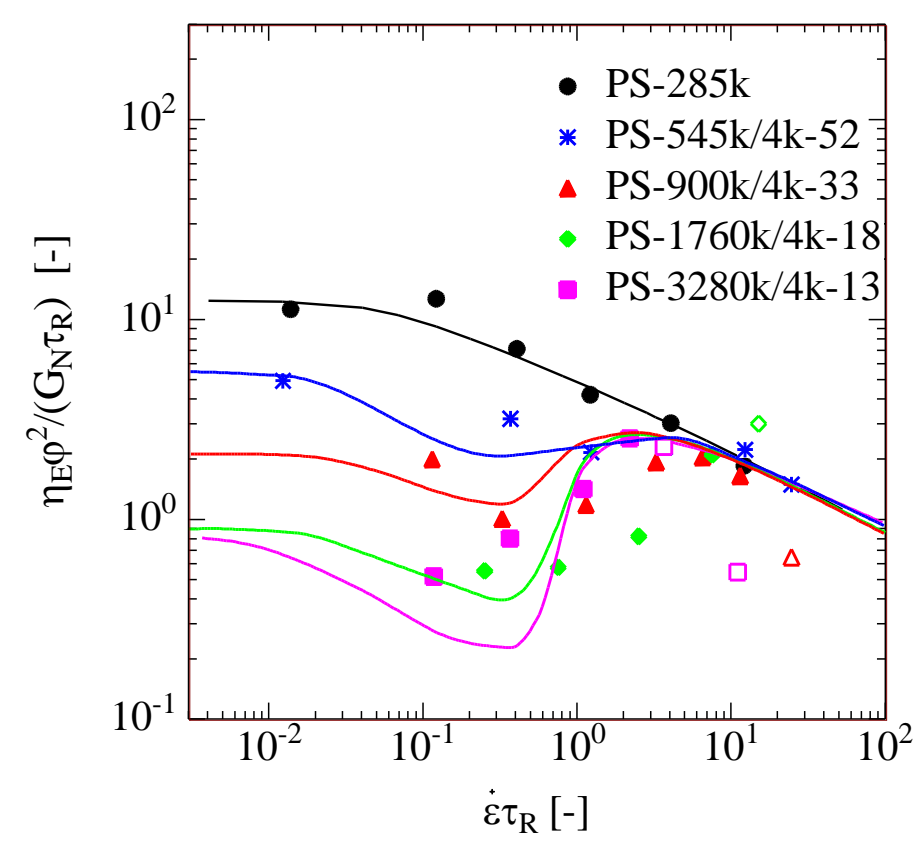

FIG 8. Dimensionless steady-state elongational viscosity $\eta_{E} \varphi^{2} /\left(G_{N} \tau_{R}\right)$ of melt PS-285k and PS/4k solutions (symbols). Lines are predictions by EIP model, Eqs. (9), (16), and (30) with $\Phi=\varphi$. Open symbols indicate samples, which fractured at the end of the test.

\subsection{PS dissolved in $2 k$ oligomeric styrene}

Oligomeric styrene $2 \mathrm{k}$ has roughly the size of a quarter of the entanglement distance in the melt and compared to oligomeric styrene $4 \mathrm{k}$ is a less effective transmitter of the interchain pressure between the test chain and the surrounding chains which act as topological constraints. We note that for oligomeric styrene $2 \mathrm{k}$ with $M_{o s}=1.92 \mathrm{~kg} / \mathrm{mol}$ (Table 1), $\Phi<\varphi$ and therefore $\tau_{a}=3 \Phi^{-4} \tau_{R}>3 \varphi^{-4} \tau_{R}$. Polymer chains dissolved in $2 \mathrm{k}$ can be stretched more than if dissolved in $4 \mathrm{k}$ before stretch relaxation via solvent molecules limits further stretch. From Eq. (31), the increase of the stretch potential at iso- $\mathrm{T}_{\mathrm{g}}$ conditions relative to the stretch of the $4 \mathrm{k}$ solutions can be expressed by a stretch enhancement factor

$$
P=\Phi^{-2} / \varphi^{-2}=\left(\varphi+(1-\varphi) \frac{M_{e m} / 4}{M_{o s}}\right)^{2}
$$

which has a value of $P=1.5$ for PS-285/2k-72, $P=1.7$ for PS-545/2k-58, and $P=2.0$ for PS285/2k-44. As seen in Figure 9, data of PS dissolved in oligomeric styrene 2k and predictions of the EIP model for both the extensional stress growth coefficient and the steady-state viscosity by use Eqs. (9), (16), and (30) with $\Phi$ given by Eq. (27) result in good agreement between data and predictions.

From Eq. (32) follows that the steady-state elongational viscosity data of all $2 \mathrm{k}$ solutions coincide with the viscosity of melts PS-285k and PS-545k for $W i_{R}=\dot{\varepsilon} \tau_{R}>>1$, if scaled in the form of $\eta_{E} \Phi^{2} /\left(G_{N} \tau_{R}\right)$. As shown in Figure 10, agreement at Weissenberg number $W i_{R} \approx 10$ is found for the viscosity data of melts and all $2 \mathrm{k}$ solutions investigated. This agreement is reached by use of the effective polymer fraction $\Phi<\varphi$, which accounts for the higher stretch 
potential of the $2 \mathrm{k}$ solution in comparison to the $4 \mathrm{k}$ solutions, and we note again that at higher elongation rates with large stretch $f$ cohesive fracture is observed as discussed in detail in [69].
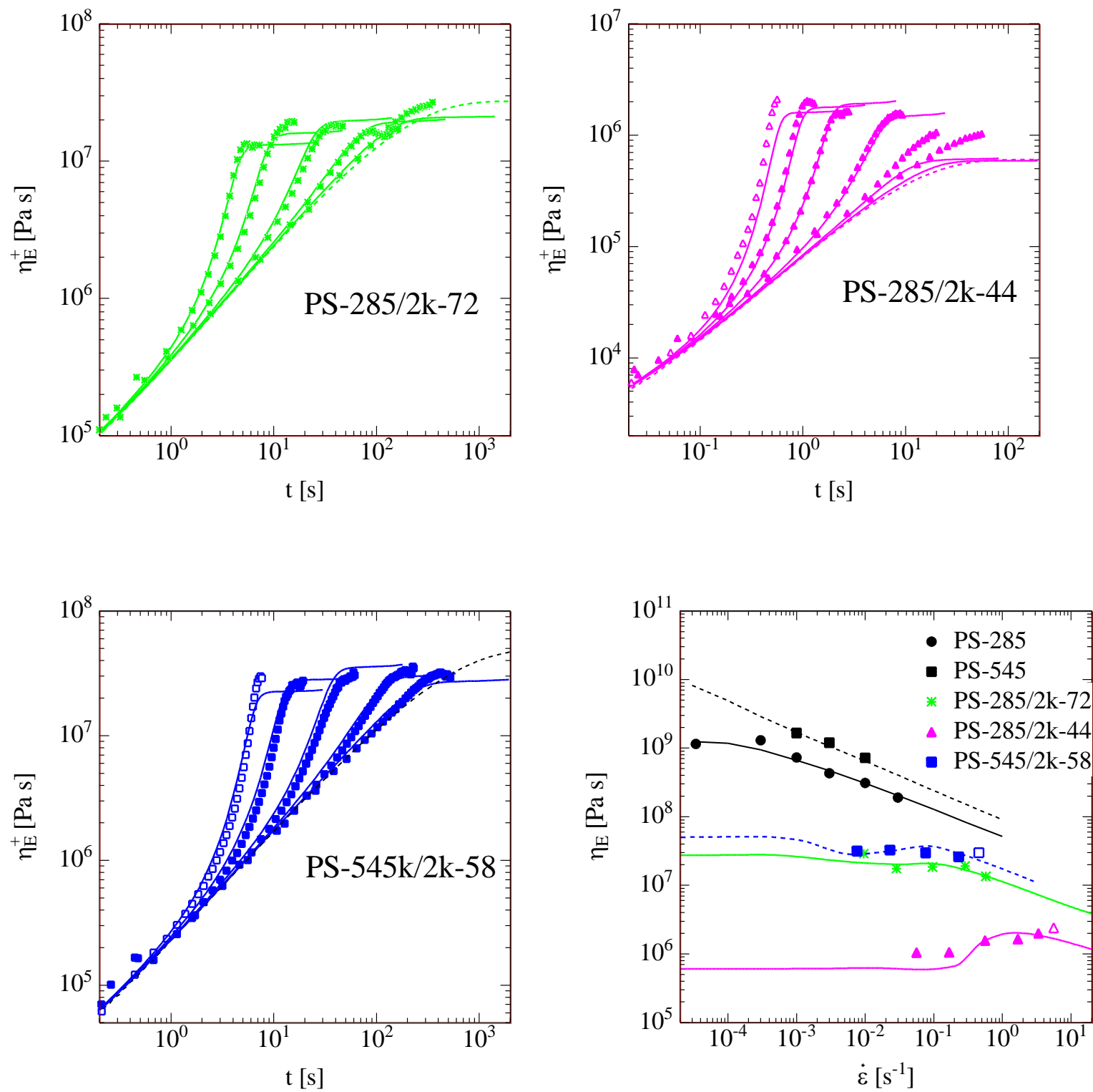

FIG 9. Data of extensional stress growth coefficient $\eta_{E}^{+}$and steady-state elongational viscosity $\eta_{E}$ (symbols) of $2 \mathrm{k}$ solutions (Table 1) and melts PS-545k and PS-285k, and predictions (lines) by EIP model, Eqs. (9), (16), (29), and (30) with $M_{o s}=1.92 \mathrm{~kg} / \mathrm{mol}$. Open symbols indicate samples, which fractured at the end of the test. 


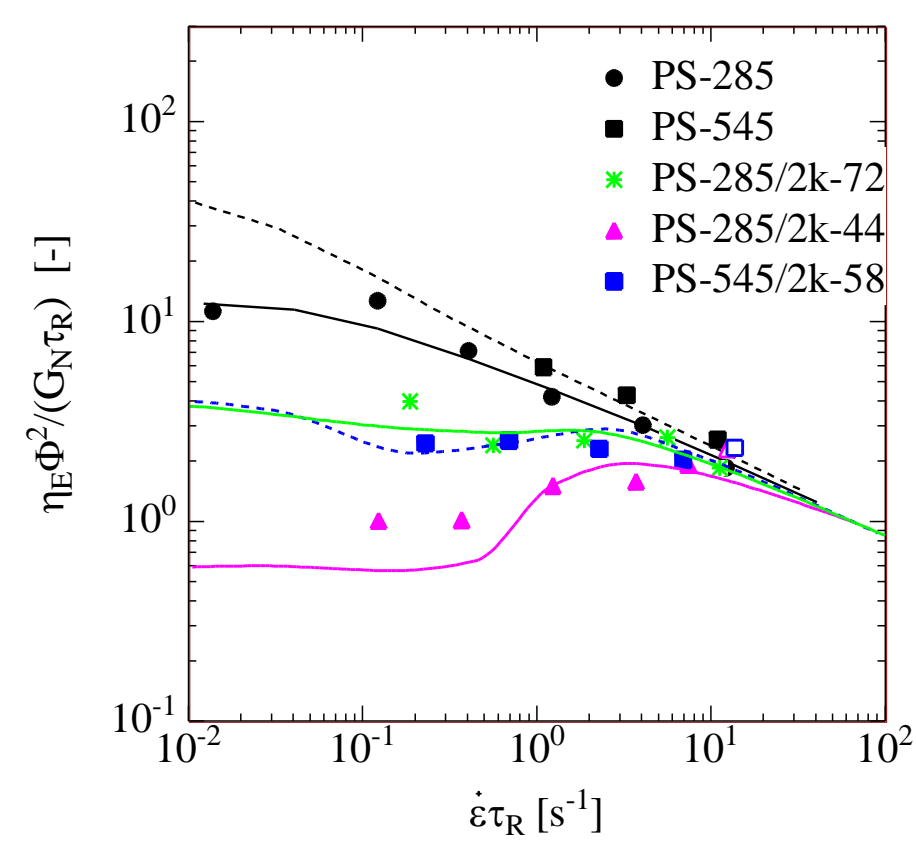

FIG 10. Dimensionless steady-state elongational viscosity $\eta_{E} \Phi^{2} /\left(G_{N} \tau_{R}\right)$ of melt PS-285k and melt PS-545k, and 2k solutions (symbols). Lines are predictions by EIP model, Eqs. (9), (16), and (30) with $\Phi$ given by Eq. (27). Open symbols indicate samples, which fractured at the end of the test.

\subsection{PS-545k dissolved in $4 k, 2 k$, and $1 k$ oligomeric styrene}

The data of the extensional stress growth coefficient of $52 \%$ PS-545k dissolved in $1 \mathrm{k}$ oligomeric styrene with $M_{o s}=0.972 \mathrm{~kg} / \mathrm{mol}$ (Table 1) and predictions of the EIP model by use Eqs. (9), (16), and (30) with $\Phi$ given by Eq. (27) are presented in Figure 11, as well as comparison of steady-state elongational viscosity data and predictions of PS-545k dissolved in oligomeric styrene of 4k (Fig. 4), 2k (Fig. 9) and 1k. The steady-state elongational viscosity of melt PS-545k is also presented. Good agreement between data of PS-545k/1k-52 and predictions is found, which validates the relation between tube diameter relaxation time and molar mass of the oligomeric solvent as expressed by Eqs. (26), (27), and (28). The stretch potential of PS-545k/1k-52 is increased by a stretch enhancement factor of $P=4.7$ (Eq. (34) ) relative to the same polymer dissolved in oligomeric solvent $4 \mathrm{k}$ at iso- $\mathrm{T}_{\mathrm{g}}$ condition.

As shown in Figure 12, the steady-state elongational viscosity data of the solutions of PS-545k in oligomeric styrene $4 \mathrm{k}, 2 \mathrm{k}$, and $1 \mathrm{k}$ coincide with the melt viscosity for $W i_{R}=\dot{\varepsilon} \tau_{R} \gg 1$, if scaled in the form of $\eta_{E} \Phi^{2} /\left(G_{N} \tau_{R}\right)$. Again, agreement is only reached by use of the effective polymer fraction $\Phi<\varphi$, which considers the higher stretch potential of the $2 \mathrm{k}$ and $1 \mathrm{k}$ solutions in comparison to the $4 \mathrm{k}$ solutions. 

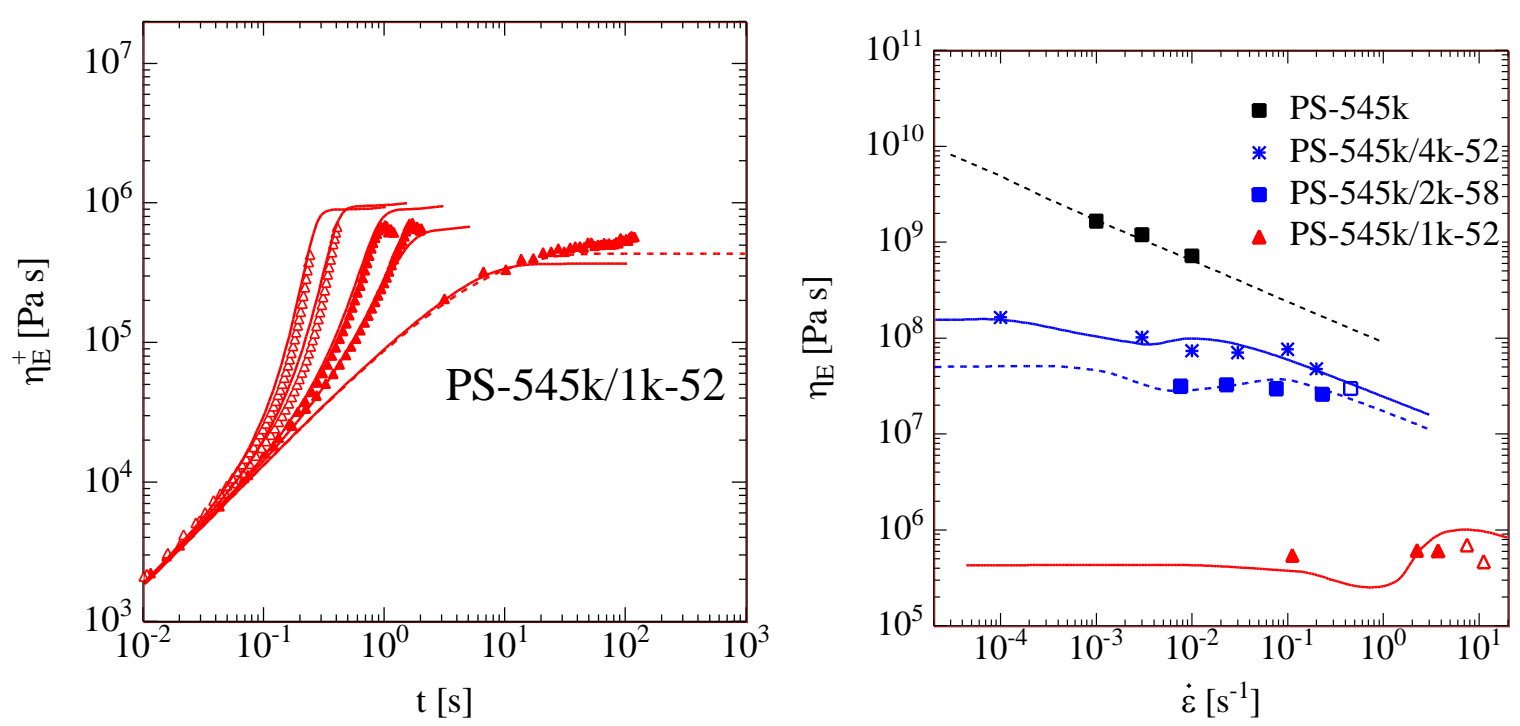

FIG 11. Data of extensional stress growth coefficient (left) and steady-state (right) elongational viscosity data (symbols) of PS-545k dissolved in $4 \mathrm{k}, 2 \mathrm{k}$, and $1 \mathrm{k}$ oligomeric styrene (Table 1) and melt PS-545k. Predictions (lines) by EIP model, Eqs. (9), (16), and (30) with $\Phi$ given by Eqs. (27) and (28). Open symbols indicate samples, which fractured at the end of the test.

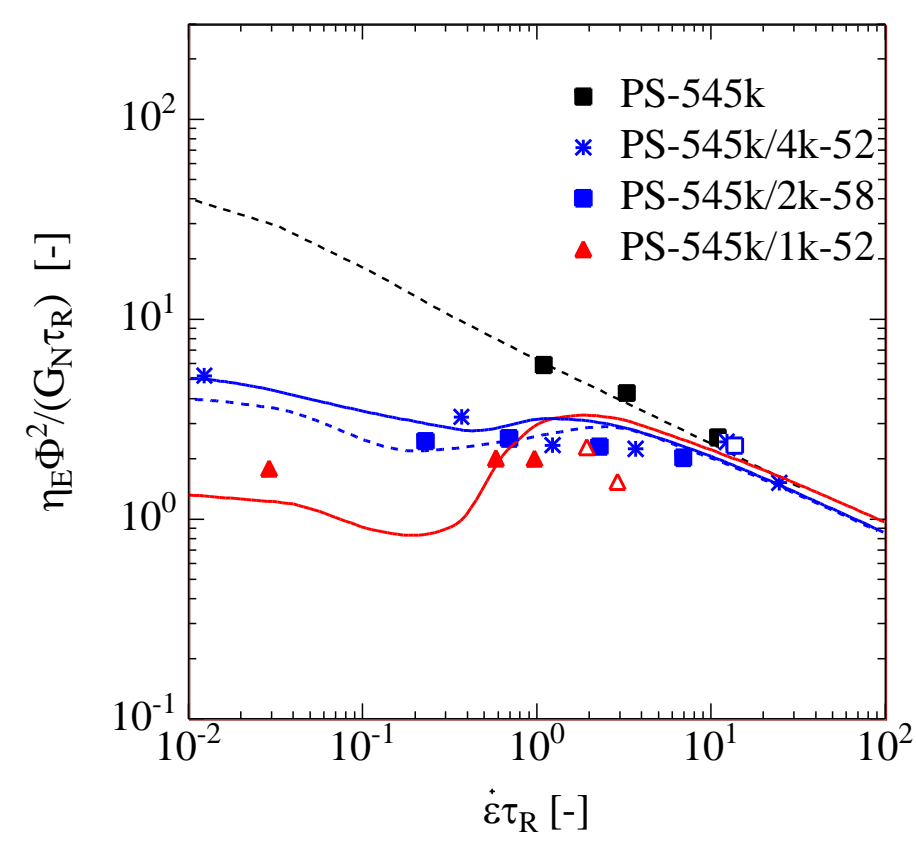

FIG 12. Dimensionless steady-state elongational viscosity $\eta_{E} \Phi^{2} /\left(G_{N} \tau_{R}\right)$ of melt PS-545k and solutions in $4 \mathrm{k}, 2 \mathrm{k}$, and $1 \mathrm{k}$ (symbols). Lines are predictions by EIP model, Eqs. (9), (16), and (30) with $\Phi$ given by Eqs. (27) and (28). Open symbols indicate samples, which fractured at the end of the test.

4.4 Entangled polystyrene solutions of high molar mass PS in diethyl phthalate (DEP) 
Due to the large stretch of the PS-545k chains in $1 \mathrm{k}$-solution at higher elongation rates, cohesive fracture occurs already at Weissenberg numbers $W i=\dot{\varepsilon} \tau_{R} \geq 2$ (for details see [69]), which limits the experimental window where a steady-state elongational viscosity can be observed (Fig. 12). For PS solutions in oligomeric styrene with molar mass $M_{o s}<1 k$, the EIP model predicts further increase of the tube diameter relaxation time (Eq. (26)) and the stretch potential according to the stretch enhancement factor $P$ (Eq. (34)), and therefore further narrowing of the experimental $\mathrm{Wi}$-window for a steady-state elongational viscosity due to cohesive fracture is expected. This is in agreement with the experimental data on solutions of 10-20\% high molar mass polystyrenes in diethyl phthalate (DEP) (Table 1) investigated by Bhattacharjee et al. (2002) [12] and Acharya et al. (2008) [45], and analysed by Wagner et al. [44]. For these solutions, only the initial decrease of the elongational viscosity corresponding to tube segment orientation with no or little stretch is observed followed by a steep upturn in the regime $W i=\dot{\varepsilon} \tau_{R} \rightarrow 1$ (Fig. 13). Predictions of the EIP model, Eqs. (9), (16), and (30) are also shown. The relaxation modulus $G(t)$ of the 10,15 , and $20 \%$ solutions of PS-1950 ( $M=1950$ $\mathrm{kg} / \mathrm{mol})$ and of the 10,15 , and $17 \%$ solutions of PS-3900 $(M=3900 \mathrm{Kg} / \mathrm{mol})$ at the measuring temperature of $21.5^{\circ} \mathrm{C}$ were taken as obtained by the scaling relations of Wagner et al. [44] (Table 2). The effective polymer fraction $\Phi$ was calculated from Eq. (27) with $M_{o s}$ replaced by the molar mass of DEP $\left(M_{D E P}=222 \mathrm{~g} / \mathrm{mol}\right)$. Data and model agree favourably in the initial extension thinning as well as in the following extension thickening regime. Due to the low molar mass of DEP and the resulting small values of $\Phi$, the tube diameter relaxation times $\tau_{a}$

(Eq. (35)) are very large for these solutions, and the limiting effect of the interchain pressure sets in only at very large stretches (indicated by the maxima of the predicted elongational viscosities), which due to cohesive fracture are outside the experimentally accessible window of a steady-state viscosity.
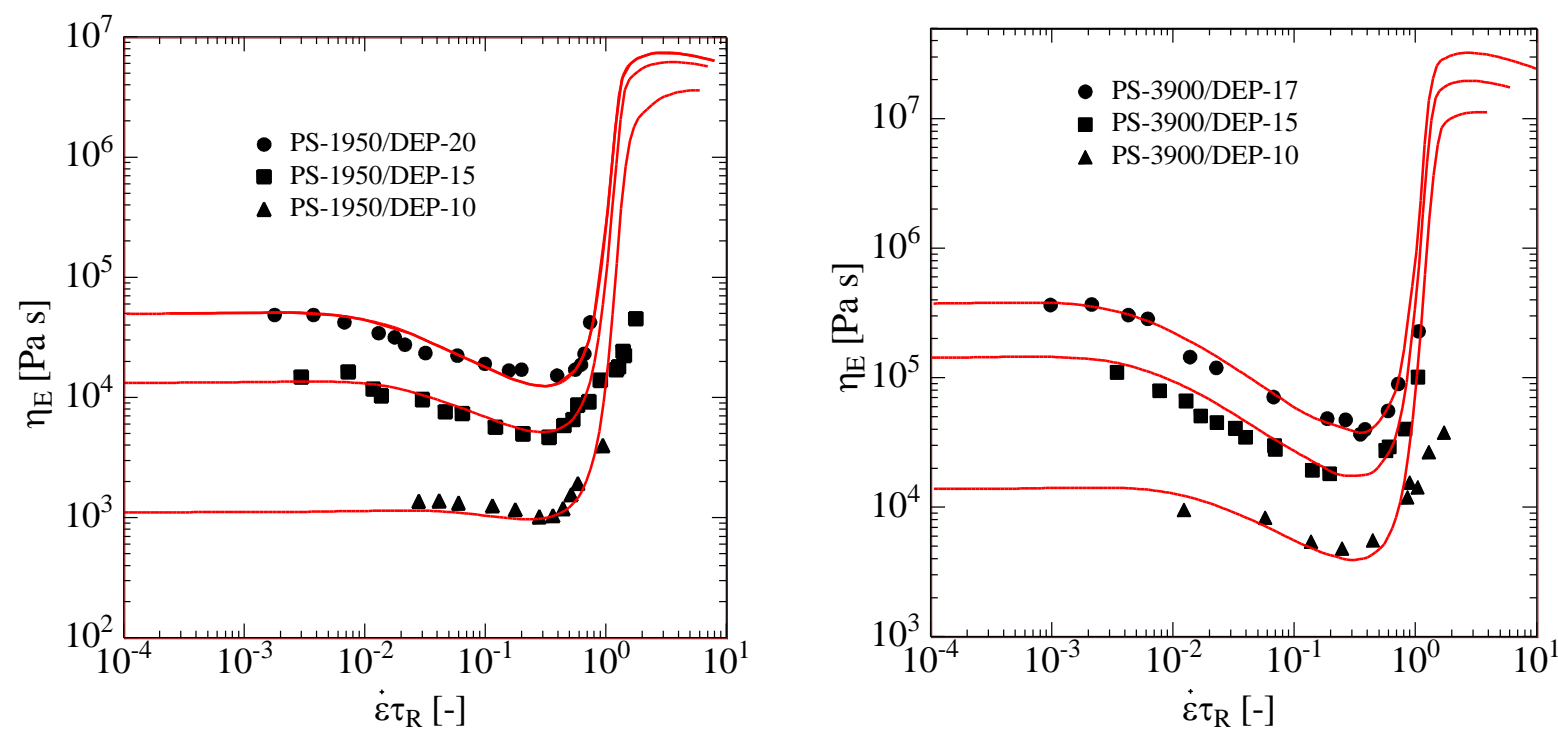

FIG 13. Steady-state elongational viscosity of 20,15 , and $10 \%$ of PS-1950 and 17,15 , and $10 \%$ of PS-3900 dissolved in DEP (Table 1). Data (symbols) from Bhattacharjee et al. (2002) [12] and Acharya et al. (2008) [45]. Lines are predictions by EIP model, Eqs. (9), (16), and (30). The effective polymer fraction $\Phi$ is given by Eq. (27) with $M_{o s}$ replaced by the molar mass of DEP $\left(M_{D E P}=222 \mathrm{~g} / \mathrm{mol}\right)$.

4.5 Stress relaxation following constant strain-rate elongation 
Huang and Rasmussen (2016) [31] analysed stress relaxation following constant strain-rate elongation of melt PS-285k and of two solutions of PS-285k in 2k oligomeric styrene, PS285k/2k-72 and PS-285k/2k-44, as well as of PS-545k dissolved in 1k oligomeric styrene, PS$545 \mathrm{k} / 1 \mathrm{k}-52$ (Table 1). They used the model of Wagner $(2014)[43,44]$, which relies on scaling relations for linear and nonlinear viscoelasticity of polystyrene melts and concentrated solutions of polystyrene in oligomeric styrene, and found good agreement between data and model. Figure 14 demonstrates that the EIP model, Eqs. (9), (16), and (30) with $\Phi$ given by Eqs. (27) and (28), results in an equally good description of these data. While for PS-285k and PS-285k/2k-72 agreement of data and model is nearly quantitative, stress relaxation at the highest elongation rates investigated occurs faster than predicted for PS-285k/2k-44 and PS$545 \mathrm{k} / 1 \mathrm{k}-52$
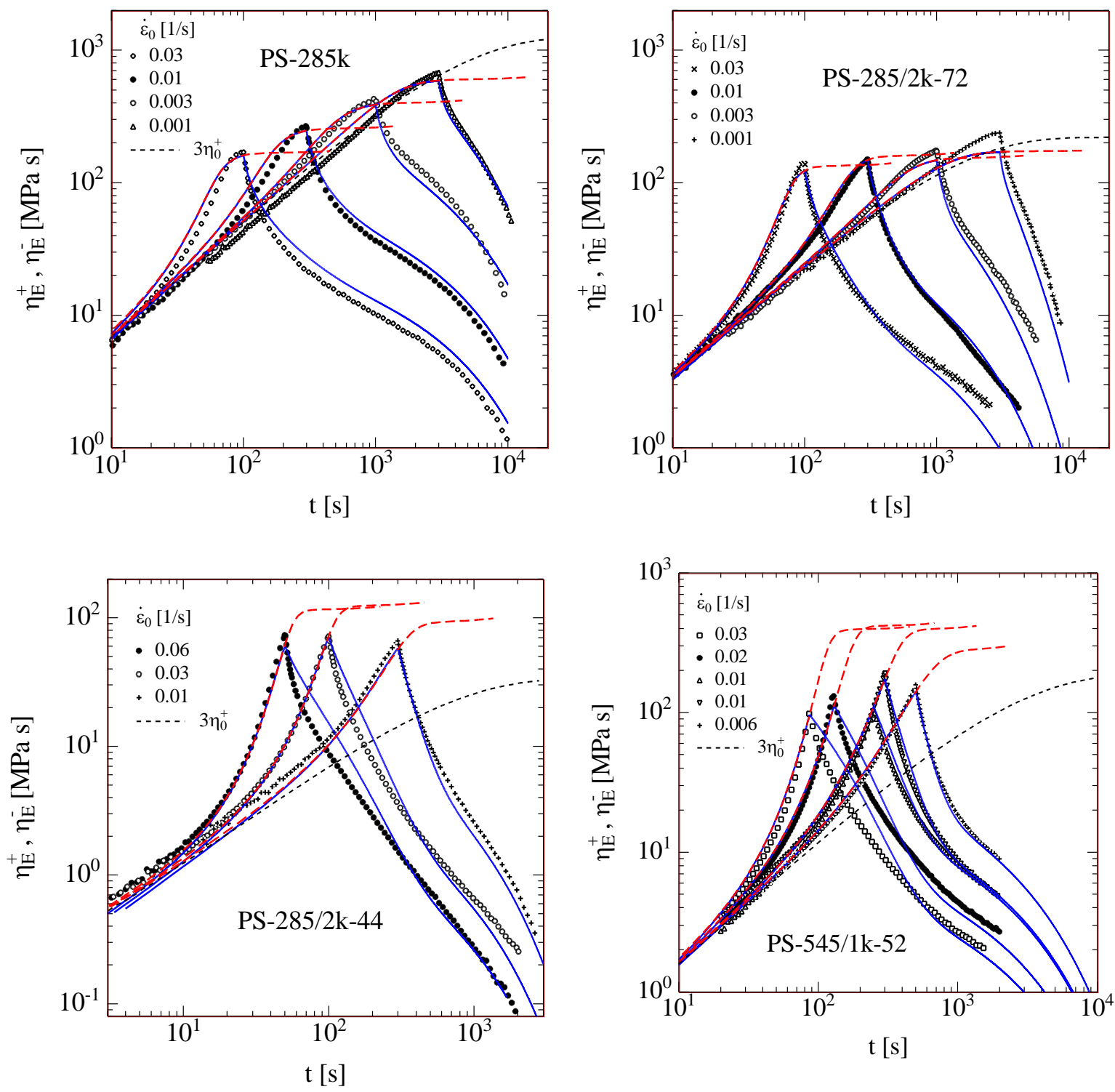

FIG 14. Data (symbols) of extensional stress growth $\left(\eta_{E}^{+}\right)$and stress relaxation coefficient ( $\eta_{E}^{-}$) of PS-285k and PS-285 dissolved in 2k oligomeric styrene, and PS-545k dissolved in 1k 
oligomeric styrene (Table 1). Predictions of $\eta_{E}^{+}$(long-dotted lines) and $\eta_{E}^{-}$(lines) by EIP model, Eqs. (9), (16), and (30) with $\Phi$ given by Eqs. (27) and (28). Dotted lines indicate linearviscoelastic extensional stress growth coefficient. 


\section{Conclusions}

We have analysed the elongational viscosity data of Bach et al. (2003) [11] and Huang et al. (2013, 2015) [7-9] on monodisperse polystyrene melts and concentrated polystyrene solutions in oligomeric styrene based on the Extended Interchain Pressure (EIP) model. The EIP model relaxes one of the basic assumptions of the original tube model of Doi and Edwards (1978) [1, 2 ], i.e. the assumption of a constant tube diameter, and assumes that chain stretch $f$ is inverse proportional to a deformation-dependent tube diameter, leading to the stress equation (16),

$$
\sigma(t)=\int_{-\infty}^{t} \frac{\partial G\left(t-t^{\prime}\right)}{\partial t^{\prime}} f^{2} \mathbf{S}_{D E}^{I A}\left(t, t^{\prime}\right) d t^{\prime}
$$

Tube diameter reduction causes interchain pressure in the lateral direction of the tube, which is proportional to the third power of stretch [41]. In addition, there is a spring force in the longitudinal direction of the tube, which is linear in stretch. This concept allows a parameterfree modelling of the elongational viscosity of monodisperse polystyrene melts [3,4], based exclusively on the linear-viscoelastic relaxation modulus $G(t)$ and the Rouse time, and without resort to friction reduction. We use the empirical BSW relaxation spectrum [46] with parameters determined by Huang et al. [7-9] for $G(t)$ here, but we note that any of the "molecular" linear-viscoelastic models relating $G(t)$ and molar mass could be used instead as long as these models allow for a quantitative description of the relaxation modulus.

When the dependence of the interchain tube pressure effect on polymer concentration and molar mass of the oligomeric solvent in the stretch evolution equation (30) is taken into account, agreement of the EIP model with the elongational benchmark data of Bach et al. (2003) [11] and Huang et al. (2013, 2015) [7-9] on monodisperse polystyrene melts and concentrated polystyrene solutions in oligomeric styrene is obtained for the investigated range from $13 \%$ to $100 \%$ (melt) polymer concentration based solely on the linear-viscoelastic relaxation modulus, polymer concentration and molar mass of the solvent. The stretch evolution $\partial f / \partial t$ (Eq. (30)) depends on 3 terms,

$$
\frac{\partial f}{\partial t}=f(\kappa: \mathbf{S})-\left(1-\frac{2}{3} \Phi^{4}\right) \frac{f-1}{\tau_{R}}-\frac{2}{3} \Phi^{4} \frac{f^{2}\left(f^{3}-1\right)}{3 \tau_{R}}
$$

These are affine deformation, Rouse relaxation with stretch relaxation Rouse time $\tau_{R}$, and the interchain pressure term with the tube diameter relaxation time, which is proportional to the Rouse time $\tau_{R}$ of the solution and to the $4^{\text {th }}$ inverse power of an effective polymer fraction $\Phi$ according to Eq. (26). The EIP model explains the effect of molar mass of the solvent on extensional thickening quantitatively: We present significant evidence that $\Phi$ is equal to the polymer fraction $\varphi$ for oligomeric styrene solvents with molar mass $M_{o s}$ equal or larger than a quarter of the entanglement molar mass, i.e. $M_{o s} \geq M_{e m} / 4=3.3 \mathrm{~kg} / \mathrm{mol}$, such as solvent $4 \mathrm{k}$. For solvents of this type, the size of the solvent molecules is equal or larger than the tube radius of the melt, and on average, the solvent molecules act laterally as short entropic springs between the central axis of the tube and the tube wall, i.e. on the length scale of the tube radius and not the tube diameter, transmitting lateral test chain fluctuations to the confining chains as effective as by direct chain-chain contacts. On the other hand, in the case of polystyrene 
dissolved in oligomeric solvents $2 \mathrm{k}$ and $1 \mathrm{k}$, the smaller solvent molecules do not span the tube radius and are therefore less effective in transmitting the lateral test chain fluctuations to the confining chains. This results in an effective polymer fraction $\Phi<\varphi$ (Eq. (27)), leading to higher stretch potentials of $2 \mathrm{k}$ and $1 \mathrm{k}$ solutions. Comparison of model predictions with the solution data of 10-20\% high molar mass polystyrenes dissolved in diethyl phthalate (DEP) (Table 1) investigated by Bhattacharjee et al. (2002) [12] and Acharya et al. (2008) [45] confirm the high stretch potential of entangled polystyrene solutions with low molar mass solvents.

Thus, with just 2 equations, stress equation (A) and stretch evolution equation (B), and without any free nonlinear-viscoelastic parameter, the EIP model predicts that at low Weissenberg numbers $W i_{R}=\dot{\varepsilon} \tau_{R}<1$, melts and solutions show extension thinning, while at $W i_{R} \cong 1$ solutions switch to extensional thickening or show a more or less constant steady-state elongational viscosity, and melts continue with extension thinning behaviour with a scaling of $\eta_{E} \propto W i_{R}^{-0.5}$. For $W i_{R} \gg>1$ and $M_{o s} \geq 3.3 \mathrm{~kg} / \mathrm{mol}$, a universal relation (33) is predicted for the steady-state elongational stress, which is independent of polymer concentration and molar mass of the solvent and depends only on the molar mass of the polymer. This universal relation agrees well with data of Nielson et al. (2008) [59] and recent data of Shahid et al. [67] as far as independency from the molar mass of the solvent is concerned, while further experimental confirmation is needed regarding the predicted independency from polymer concentration. Future work will also show whether the EIP model can be extended to melts and solutions with other chemistries as investigated e.g. by Sridhar et al. (2014) [70] and Morelly et al. (2019) [71]. 


\section{References}

[1] Doi, M., and Edwards, S. F., "Dynamics of concentrated polymer systems. Part 2. Molecular motion under flow,' J. Chem. Soc., Faraday Transactions 2: Molecular and Chemical Physics 74, 1802-1817 (1978).

[2] Doi, M., and Edwards, S. F., "Dynamics of concentrated polymer systems. Part 3. - The constitutive equation,'” J. Chem. Soc., Faraday Transactions 2: Molecular and Chemical Physics 74, 1818-1832 (1978).

[3] Wagner, M. H., and Rolón-Garrido, V. H., "Recent advances in constitutive modeling of polymer melts. novel trends of rheology III," AIP Conf. Proc. 1152, 16-31 (2009).

[4] Wagner, M. H., and Rolón-Garrido, V. H., "Nonlinear rheology of linear polymer melts: Modeling chain stretch by interchain tube pressure and Rouse time,' Korea Australia Rheol. J. 21, 203-211 (2009).

[5] Doi, M., and Edwards, S. F., The Theory of Polymer Dynamics (Oxford University Press, Oxford, 1986).

[6] Likhtman, A. E., and McLeish, T. C., "Quantitative theory for linear dynamics of linear entangled polymers,'” Macromolecules 35, 6332-6343 (2002).

[7] Huang, Q., Alvarez, N. J., Matsumiya, Y., Rasmussen, H. K., Watanabe, H., and Hassager, O., "Extensional rheology of entangled polystyrene solutions suggests importance of nematic interactions," ACS Macro Letters 2, 741-744 (2013)

[8] Huang, Q., Hengeller, L., Alvarez, N. J., and Hassager, O., "'Bridging the gap between polymer melts and solutions in extensional rheology," Macromolecules 48, 4158-4163 (2015).

[9] Huang, Q., Mednova, O., Rasmussen, H. K., Alvarez, N. J., Skov, A. L., Almdal, K., and Hassager, O., "Concentrated polymer solutions are different from melts: Role of entanglement molecular weight,' Macromolecules 46, 5026-5035 (2013).

[10] Costanzo, S., Huang, Q., Ianniruberto, G., Marrucci, G., Hassager, O., and Vlassopoulos, D., "Shear and extensional rheology of polystyrene melts and solutions with the same number of entanglements," Macromolecules 49, 3925-3935 (2016).

[11] Bach, A., Almdal, K., Rasmussen, H. K., and Hassager, O., "Elongational viscosity of narrow molar mass distribution polystyrene,'” Macromolecules 36, 5174-5179 (2003).

[12] Bhattacharjee, P. K., Oberhauser, J. P., McKinley, G. H., Leal, L. G., and Sridhar, T., "Extensional rheometry of entangled solutions,"' Macromolecules 35, 10131-10148 (2002). [13] Ye, X., Larson, R. G., Pattamaprom, C., and Sridhar, T., "Extensional properties of monodisperse and bidisperse polystyrene solutions," J. Rheol. 47, 443-468 (2003).

[14] Ianniruberto, G., Brasiello, A., and Marrucci, G., "'Simulations of fast shear flows of PS oligomers confirm monomeric friction reduction in fast elongational flows of monodisperse PS melts as indicated by rheooptical data,' Macromolecules 45, 8058-8066 (2012).

[15] Ianniruberto, G., Brasiello, A., and Marrucci, G., "Friction coefficient does not stay constant in nonlinear viscoelasticity," 2011.

[16] Yaoita, T., Isaki, T., Masubuchi, Y., Watanabe, H., Ianniruberto, G., and Marrucci, G., "Primitive chain network simulation of elongational flows of entangled linear chains: stretch/orientation-induced reduction of monomeric friction," Macromolecules 45, 27732782 (2012).

[17] Giesekus, H., "Constitutive equations for polymer fluids based on the concept of configuration-dependent molecular mobility: A generalized mean-configuration model,' J. Non-Newtonian Fluid Mech. 17, 349-372 (1985).

[18] Wiest, J., 'A differential constitutive equation for polymer melts,'” Rheol. Acta 28, 4-12 (1989). 
[19] Gupta, R. K., Nguyen, D. A., and Sridhar, T., "'Extensional viscosity of dilute polystyrene solutions: Effect of concentration and molecular weight,' Physics of Fluids 12, 1296-1318 (2000).

[20] Park, J., Mead, D. W., and Denn, M. M., "Stochastic simulation of entangled polymeric liquids in fast flows: Microstructure modification," J. Rheol. 56, 1057-1081 (2012).

[21] Masubuchi, Y., Yaoita, T., Matsumiya, Y., Watanabe, H., Ianniruberto, G., and Marrucci, G., "Stretch/orientation induced acceleration in stress relaxation in coarse-grained molecular dynamics simulations," Nihon Reoroji Gakkaishi 41, 35-37 (2013).

[22] Desai, P. S., and Larson, R. G., " Constitutive model that shows extension thickening for entangled solutions and extension thinning for melts,' J. Rheol. 58, 255-279 (2014).

[23] Marrucci, G., and Grizzuti, N., "Fast flows of concentrated polymers: predictions of the tube model on chain stretching," Gazz. Chim. Itali. 118, 179-185 (1988).

[24] Messé, L., and Prud'Homme, R. E., "'Orientation and relaxation study of polystyrene: Polystyrene/poly (phenylene oxide) blends,' J. Poly. Sci. Part B: Poly. Phys. 38, 1405-1415 (2000).

[25] Thulstrup, E. W., and Michl, J., "'Orientation and linear dichroism of symmetrical aromatic molecules imbedded in stretched polyethylene," J. American Chem. Soc. 104 5594-5604 (1982)

[26] Likhtman, A. E., "Whither tube theory: from believing to measuring," J. NonNewtonian Fluid Mech. 157, 158-161 (2009).

[27] Doi, M., Pearson, D., Kornfield, J., and Fuller, G., "Effect of nematic interaction in the orientational relaxation of polymer melts," Macromolecules 22, 1488-1490 (1989).

[28] Doi, M., Introduction to polymer physics (Oxford university press, 1996).

[29] Graf, R., Heuer, A., and Spiess, H. W., "Chain-Order Effects in Polymer Melts Probed by H 1 Double-Quantum NMR Spectroscopy,’’ Phys. Rev. Letters 80, 5738 (1998).

[30] Rubinstein, M., and Colby, R. H., Polymer physics (Oxford University Press New York, 2003).

[31] Huang, Q., and Rasmussen, H. K., "'Stress relaxation following uniaxial extension of polystyrene melt and oligomer dilutions,"' J. Rheol. 60, 465-471 (2016).

[32] Ianniruberto, G., " Extensional flows of solutions of entangled polymers confirm reduction of friction coefficient," Macromolecules 48, 6306-6312 (2015).

[33] Park, G. W., and Ianniruberto, G., "Flow-induced nematic interaction and friction reduction successfully describe ps melt and solution data in extension startup and relaxation," Macromolecules 50, 4787-4796 (2017).

[34] Matsumiya, Y., Watanabe, H., Masubuchi, Y., Huang, Q., and Hassager, O., " Nonlinear Elongational Rheology of Unentangled Polystyrene and Poly (p-tert-butylstyrene) Melts,', Macromolecules 51, 9710-9729 (2018).

[35] Ianniruberto, G., Brasiello, A., and Marrucci, G., "Modeling Unentangled Polystyrene Melts in Fast Elongational Flows," Macromolecules (2019).

[36] O’Connor, T. C., Hopkins, A., and Robbins, M. O., 'Stress Relaxation in Highly Oriented Melts of Entangled Polymers,'” Macromolecules (2019).

[37] Pearson, D. S., Kiss, A. D., Fetters, L. J., and Doi, M., 'Flow-induced birefringence of concentrated polyisoprene solutions,"' J. Rheol. 33, 517-535 (1989).

[38] Ianniruberto, G., and Marrucci, G., "'On compatibility of the Cox-Merz rule with the model of Doi and Edwards," J. Non-Newtonian Fluid Mech. 65, 241-246 (1996).

[39] Rolón-Garrido, V. H., "The molecular stress function (MSF) model in rheology," Rheol. Acta 53, 663-700 (2014).

[40] Narimissa, E., and Wagner, M. H., "Review of the hierarchical multi-mode molecular stress function model for broadly distributed linear and LCB polymer melts," Poly. Eng. \& Sci. 59, 573-583 (2018). 
[41] Marrucci, G., and Ianniruberto, G., “'Interchain pressure effect in extensional flows of entangled polymer melts," Macromolecules 37, 3934-3942 (2004).

[42] Wagner, M. H., Kheirandish, S., and Hassager, O., "Quantitative prediction of transient and steady-state elongational viscosity of nearly monodisperse polystyrene melts,' J. Rheol. 49, 1317-1327 (2005).

[43] Wagner, M. H., 'Scaling relations for elongational flow of polystyrene melts and concentrated solutions of polystyrene in oligomeric styrene,'” Rheol. Acta 53, 765-777 (2014).

[44] Wagner, M. H., Narimissa, E., and Rolón-Garrido, V. H., "From melt to solution: Scaling relations for concentrated polystyrene solutions,' J. Rheol. 59, 1113-1130 (2015).

[45] Acharya, M., Bhattacharjee, P., Nguyen, D., and Sridhar, T., "Are entangled polymeric solutions different from melts?," 2008.

[46] Baumgaertel, M., Schausberger, A., and Winter, H. H., "The relaxation of polymers with linear flexible chains of uniform length," Rheol. Acta 29, 400-408 (1990).

[47] Larson, R., "Combinatorial rheology of branched polymer melts," Macromolecules 34, 4556-4571 (2001).

[48] Park, S. J., and Larson, R. G., "'Dilution exponent in the dynamic dilution theory for polymer melts,' J. Rheol. 47, 199-211 (2003).

[49] Auhl, D., Chambon, P., McLeish, T. C. B., and Read, D. J., “Elongational Flow of Blends of Long and Short Polymers: Effective Stretch Relaxation Time,'” Phys. Rev. Letters 103 (2009).

[50] Watanabe, H., Ishida, S., Matsumiya, Y., and Inoue, T., "Test of full and partial tube dilation pictures in entangled blends of linear polyisoprenes," Macromolecules 37, 66196631 (2004).

[51] Osaki, K., Nishizawa, K., and Kurata, M., "Material time constant characterizing the nonlinear viscoelasticity of entangled polymeric systems,' Macromolecules 15, 1068-1071 (1982).

[52] Takahashi, M., Isaki, T., Takigawa, T., and Masuda, T., "'Measurement of biaxial and uniaxial extensional flow behavior of polymer melts at constant strain rates," J. Rheol. 37, 827-846 (1993).

[53] Isaki, T., Takahashi, M., and Urakawa, O., "Biaxial damping function of entangled monodisperse polystyrene melts: Comparison with the Mead-Larson-Doi model,' J. Rheol. 47, 1201-1210 (2003).

[54] Menezes, E., and Graessley, W., " Nonlinear rheological behavior of polymer systems for several shear-flow histories,” J. Poly. Sci. Part B: Poly. Phys. 20, 1817-1833 (1982). [55] Ferry, J. D., Viscoelastic Properties of Polymers (John Wiley and Sons, New York, 1980).

[56] Luap, C., Müller, C., Schweizer, T., and Venerus, D. C., "Simultaneous stress and birefringence measurements during uniaxial elongation of polystyrene melts with narrow molecular weight distribution,"' Rheol. Acta 45, 83-91 (2005).

[57] McLeish, T. C. B., and Larson, R. G., "Molecular constitutive equations for a class of branched polymers: The pom-pom polymer,' J. Rheol. 42, 81-110 (1998).

[58] Mead, D. W., Yavich, D., and Leal, L. G., "'The reptation model with segmental stretch - II. Steady flow properties,' Rheol. Acta 34, 360-383 (1995).

[59] Nielsen, J. K., Rasmussen, H. K., and Hassager, O., "'Stress relaxation of narrow molar mass distribution polystyrene following uniaxial extension," J. Rheol. 52, 885-899 (2008). [60] Marrucci, G., and de Cindio, B., "The stress relaxation of molten PMMA at large deformations and its theoretical interpretation," Rheol. Acta 19, 68-75 (1980).

[61] Wagner, M., and Schaeffer, J., "Nonlinear strain measures for general biaxial extension of polymer melts," J. Rheol. 36, 1-26 (1992). 
[62] Wagner, M. H., and Schaeffer, J., "Rubbers and polymer melts: Universal aspects of nonlinear stress-strain relations," J. Rheol. 37, 643-661 (1993).

[63] Wagner, M. H., and Schaeffer, J., "Assessment of nonlinear strain measures for extensional and shearing flows of polymer melts," Rheol. Acta 33, 506-516 (1994). [64] Wagner, M. H., Rubio, P., and Bastian, H., "The molecular stress function model for polydisperse polymer melts with dissipative convective constraint release,' J. Rheol. 45, 1387-1412 (2001)

[65] Wagner, M. H., "An extended interchain tube pressure model for elongational flow of polystyrene melts and concentrated solutions,' J. Non-Newtonian Fluid Mech. 222, 121-131 (2015).

[66] O'Connor, T. C., Alvarez, N. J., and Robbins, M. O., "'Relating chain conformations to extensional stress in entangled polymer melts,' Physical review letters 121, 047801 (2018)

[67] Shahid, T., Clasen, C., Oosterlinck, F., and van Ruymbeke, E., 'Diluting Entangled Polymers Affects Transient Hardening but Not Their Steady Elongational Viscosity," Macromolecules (2019).

[68] Wagner, M. H., Rolón-Garrido, V. H., Nielsen, J. K., Rasmussen, H. K., and Hassager, O., "A constitutive analysis of transient and steady-state elongational viscosities of bidisperse polystyrene blends," J. Rheol. 52, 67-86 (2008).

[69] Wagner, M. H., Narimissa, E., and Huang, Q., ' On the origin of brittle fracture of entangled polymer solutions and melts,'” J. Rheol. 62, 221-233 (2018).

[70] Sridhar, T., Acharya, M., Nguyen, D. A., and Bhattacharjee, P. K., " On the extensional rheology of polymer melts and concentrated solutions," Macromolecules 47, 379-386 (2014).

[71] Morelly, S. L., Palmese, L., Watanabe, H., and Alvarez, N. J., " Effect of finite extensibility on nonlinear extensional rheology of polymer melts," Macromolecules 52, 915$922(2019)$ 